\title{
THE RIEMANN ZETA-FUNCTION AND THE ONE-DIMENSIONAL WEYL-BERRY CONJECTURE FOR FRACTAL DRUMS
}

\author{
MICHEL L. LAPIDUS and CARL POMERANCE
}

[Received 7 April 1991-Revised 10 December 1991]

\begin{abstract}
Based on his earlier work on the vibrations of 'drums with fractal boundary', the first author has refined M. V. Berry's conjecture that extended from the 'smooth' to the 'fractal' case H. Weyl's conjecture for the asymptotics of the eigenvalues of the Laplacian on a bounded open subset of $\mathbb{R}^{n}$ (see [16]). We solve here in the one-dimensional case (that is, when $n=1$ ) this 'modified Weyl-Berry conjecture'. We discover, in the process, some unexpected and intriguing connections between spectral geometry, fractal geometry and the Riemann zeta-function. We therefore show that one can 'hear' (that is, recover from the spectrum) not only the Minkowski fractal dimension of the boundary - as was established previously by the first author-but also, under the stronger assumptions of the conjecture, its Minkowski content (a 'fractal' analogue of its 'length').

We also prove (still in dimension one) a related conjecture of the first author, as well as its converse, which characterizes the situation when the error estimates of the aforementioned paper are sharp.
\end{abstract}

\section{Introduction}

Let $\Omega$ be an open set in $\mathbb{R}^{n}(n \geqslant 1)$, with boundary $\Gamma=\partial \Omega$. We assume that $\Omega$ is non-empty and bounded (or, more generally, of finite volume), but otherwise arbitrary. We consider the following eigenvalue problem:

$$
\left\{\begin{aligned}
-\Delta u & =\lambda u \text { in } \Omega, \\
u & =0 \text { on } \Gamma,
\end{aligned}\right.
$$

where $\Delta=\sum_{k=1}^{n} \partial^{2} / \partial x_{k}^{2}$ denotes the Dirichlet Laplacian on $\Omega$.

In this general setting, the problem $(\mathrm{P})$ is interpreted in the variational sense. More precisely, the scalar $\lambda$ is said to be an eigenvalue of $(\mathrm{P})$ if there exists $u \neq 0$ in $H_{0}^{1}(\Omega)$ (the closure of $C_{0}^{\infty}(\Omega)$, the space of smooth functions with compact support contained in $\Omega$, in the Sobolev space $H^{1}(\Omega)$ ) satisfying equation (1.1) in the distributional sense. (See, for example, [23; 28, § II; 16, § 2.2].)

As is well-known, the spectrum of (P) is discrete and consists of a sequence $\left(\lambda_{r}\right)_{r=1}^{\infty}$ of eigenvalues (with finite multiplicity) written in increasing order according to their multiplicity:

$$
0<\lambda_{1} \leqslant \lambda_{2} \leqslant \ldots \leqslant \lambda_{r} \leqslant \ldots, \quad \text { with } \lambda_{r} \rightarrow+\infty \text { as } r \rightarrow \infty \text {. }
$$

Let $N(\lambda)$ denote the (eigenvalue) 'counting function' of $(\mathrm{P})$; that is, for $\lambda>0$,

$$
N(\lambda)=\#\left\{r \geqslant 1: \lambda_{r} \leqslant \lambda\right\}
$$

is the number of eigenvalues of $(\mathrm{P})$ up to $\lambda$, counted according to multiplicity.

The research of the first author was partially supported by the National Science Foundation under Grants DMS-8703138, DMS-8904389 and DMS-9207098, as well as by the M. G. Michael Award for Excellence in Research from the Franklin College of Arts and Sciences of the University of Georgia. The research of the second author was partially supported by the National Science Foundation under Grant DMS-8803297.

1991 Mathematics Subject Classification: 11M06, 28A12, 34B24.

Proc. London Math. Soc. (3) 66 (1993) 41-69. 
Generalizing $\mathrm{H}$. Weyl's classical theorem, G. Métivier [28] showed that

$$
N(\lambda)=(1+o(1)) \varphi(\lambda), \quad \text { as } \lambda \rightarrow+\infty,
$$

where the 'Weyl term' $\varphi(\lambda)$ is given by

$$
\varphi(\lambda)=(2 \pi)^{-n} \mathscr{B}_{n}|\Omega|_{n} \lambda^{n / 2} .
$$

Here, $|A|_{n}$ denotes the $n$-dimensional Lebesgue measure or 'volume' of $A \subset \mathbb{R}^{n}$ and $\mathscr{B}_{n}$ is the volume of the unit ball in $\mathbb{R}^{n}$.

It is natural to wonder whether the asymptotic expansion of $N(\lambda)$ admits a second term. In the 'smooth' case, this was conjectured by $\mathrm{H}$. Weyl [35] as follows. If $\Gamma=\partial \Omega$ is 'sufficiently regular', then

$$
N(\lambda)=\varphi(\lambda)-\mathscr{C}_{n}|\Gamma|_{n-1} \lambda^{(n-1) / 2}+o\left(\lambda^{(n-1) / 2}\right), \quad \text { as } \lambda \rightarrow+\infty,
$$

where $\mathscr{C}_{n}$ is a positive constant depending only on $n$. Here, $|\Gamma|_{n-1}$ denotes the $(n-1)$-dimensional volume of $\Gamma$; for example, the length of $\Gamma \subset \mathbb{R}^{2}$ if $n=2$.

An important step on the way to Weyl's conjecture was made by R. T. Seeley $[30,31]$, and then, when $n \geqslant 4$, by Pham The Lai [29]. Extending, in particular, earlier work of L. Hörmander [11], they showed that if $\Gamma$ is 'smooth' (that is, of class $C^{\infty}$ ), then the following sharp remainder estimate holds:

$$
N(\lambda)=\varphi(\lambda)+O\left(\lambda^{(n-1) / 2}\right), \quad \text { as } \lambda \rightarrow+\infty .
$$

Further, V. Ja. Ivrii $[\mathbf{1 3}, \mathbf{1 4}]$ has established (1.6) under an additional assumption (roughly, that the set of 'multiply reflected periodic geodesics in $\bar{\Omega}$ is of measure zero'). Therefore, under these hypotheses, the conclusion of Weyl's conjecture is true. (For related results, see also R. B. Melrose's work [26, 27]; a synthesis of the methods of Ivrii and Melrose is provided in [12].)

What happens if the boundary is 'non-smooth'? The physicist M. V. Berry $[1,2]$-motivated in part by the study of the scattering of waves from rough surfaces and the study of porous media-conjectured that if $\Gamma$ is 'fractal', then

$$
N(\lambda)=\varphi(\lambda)-\mathscr{C}_{n, H} \mathscr{H}(H ; \Gamma) \lambda^{H / 2}+o\left(\lambda^{H / 2}\right), \quad \text { as } \lambda \rightarrow+\infty,
$$

where $\mathscr{C}_{n, H}$ is a positive constant depending only on $n$ and $H$. Here, $H$ denotes the Hausdorff dimension of the boundary $\Gamma$ and $\mathscr{H}(H ; \Gamma)$ the (normalized) $H$-dimensional Hausdorff measure of $\Gamma$. (Of course, if $\Gamma$ is 'smooth', say of class $C^{1}$, then $H=n-1$ and (at least formally) we recover (1.6) from (1.8).) Berry [1, Equation 2, p. 51], by analogy with the 'smooth' case, even stated an explicit value for $\mathscr{C}_{n, H}$; namely,

$$
\mathscr{C}_{n, H}=\left(4(4 \pi)^{H / 2} \Gamma\left(1+\frac{1}{2} H\right)\right)^{-1},
$$

where $\Gamma(s)$ denotes the classical gamma function.

Unfortunately, Berry's beautiful conjecture has turned out to be false. In an important paper [6], J. Brossard and R. Carmona disproved Berry's conjecture and suggested that the Minkowski dimension was more appropriate than the Hausdorff dimension to measure the 'roughness' of the boundary $\Gamma$. In fact, under suitable hypotheses, they obtained one- and two-sided estimates for the second term (expressed in terms of the Minkowski dimension) in the asymptotic expansion of the 'partition function' $Z(t)=\sum_{r=1}^{\infty} e^{-\lambda, t}$, a well-known 'smoothing' of the 'counting function' $N(\lambda)$. Although it is known how, via Tauberian theory, to get the asymptotics for the second term for $Z(t)$ from such a result for $N(\lambda)$, no known Tauberian theorem allows us to go in the other direction. 
A specific reformulation of Berry's conjecture on $N(\lambda)$, called the 'modified Weyl-Berry conjecture', was made by the first author in [16]. In order to state it precisely, we first discuss the, perhaps not so well-known, concepts of Minkowski dimension and measurability.

Let $D=D(\Gamma)$ denote the Minkowski dimension of $\Gamma$. Recall that $D$ is the infimum of the numbers $d \geqslant 0$ such that the (d-dimensional) upper Minkowski content

$$
\mathcal{M}^{*}(d ; \Gamma):=\limsup _{\varepsilon \rightarrow 0^{+}} \varepsilon^{-(n-d)}\left|\Gamma_{\varepsilon} \cap \Omega\right|_{n}<+\infty,
$$

where $\Gamma_{\varepsilon}$, the $\varepsilon$-neighbourhood of $\Gamma$, is the set of $x \in \mathbb{R}^{n}$ within a distance less than $\varepsilon$ from $\Gamma$; moreover, $\mathcal{M}_{*}(d ; \Gamma)$, the (d-dimensional) lower Minkowski content of $\Gamma$, is defined just as $\mathcal{M}^{*}(d ; \Gamma)$, but by means of the lower limit rather than the upper limit in (1.10). We say that $\Gamma$ is Minkowski measurable if $0<\mathcal{M}_{*}(D ; \Gamma)=\mathcal{M}^{*}(D ; \Gamma)<+\infty$ and then call this common number $\mathcal{M}(D ; \Gamma)$, the Minkowski content of $\Gamma$. (Note that these definitions not only involve $\Gamma$, but the set $\Omega$ as well.)

The larger $D$ is, the more irregular $\Gamma$ is. The (easy) fact that $D=D(\Gamma) \in$ $[n-1, n]$ since $\Gamma=\partial \Omega$, with $\Omega$ open and non-dense in $\mathbb{R}^{n}$, is proved in [16, Corollary 3.2]. Following [16], we say that $\Gamma$ is 'fractal' if $D \in(n-1, n]$, and 'non-fractal' otherwise (that is, if $D=n-1$, the topological dimension of $\Gamma$ ). We stress that no assumption of self-similarity, in the sense of [24], is made about $\Gamma$.

The real number $D$, also called 'entropy' or 'box' dimension in the literature, was first extensively studied by G. Bouligand in [4]. For an equivalent definition and further properties of $D$, see, for example, $[16, \S 3]$, especially Corollary 3.1 , and $[7,8,9,18,25,34]$. We refer to $[3,10,15,33]$ for specific properties and alternative definitions of $D$ when $n=1$, which is the case of most interest to us in this paper.

We now recall the statement of the 'modified Weyl-Berry (in short, MWB) conjecture' [16, Conjecture 5.1, $\$ 5.2$, p. 520], for the Dirichlet problem:

Conjecture 1 (modified Weyl-Berry conjecture). If $\Omega$ has 'fractal' boundary $\Gamma$ with Minkowski dimension $D \in(n-1, n)$ and if $\Gamma$ is 'Minkowski measurable', then

$$
N(\lambda)=\varphi(\lambda)-c_{n, D} \mathcal{M}(D ; \Gamma) \lambda^{D / 2}+o\left(\lambda^{D / 2}\right), \quad \text { as } \lambda \rightarrow+\infty,
$$

where $c_{n, D}$ is a positive constant depending only on $n$ and $D$.

In [16, Theorem 2.1, Corollary 2.1, and Theorem 2.3, pp. 479-480 and 482-483], M. L. Lapidus has partially solved a more general form of this conjecture by proving, in particular, that if $\Gamma$ is 'fractal' with $\mathcal{M}^{*}(D ; \Gamma)<+\infty$, then Weyl's asymptotic law with error term holds:

$$
N(\lambda)=\varphi(\lambda)+O\left(\lambda^{D / 2}\right), \quad \text { as } \lambda \rightarrow+\infty .
$$

(Also see [20].)

Moreover, the first author has shown by means of examples that the remainder estimate in (1.12) is sharp in every possible 'fractal' (that is, Minkowski) dimension $D \in(n-1, n)$. (See [16, Example 5.1, pp. 512-514] when $n=1$, and its generalization to $n \geqslant 1$ [16, Example 5.1', pp. 514-515].) 
In this paper, we shall prove Conjecture 1 (the 'MWB conjecture') when $n=1$. (See Corollary 2.3 below.) In the process, we shall establish some unexpected and intriguing connections with the Riemann zeta-function. Indeed, we shall show that the constant $c_{1, D}$ occurring in (1.11) is given by

$$
c_{1, D}=2^{-(1-D)} \pi^{-D}(1-D)(-\zeta(D)),
$$

where $\zeta=\zeta(s)$ denotes the Riemann zeta-function. (Since $n=1$, we have $D \in(0,1)$ and thus $\zeta(D)<0$, so that $c_{1, D}>0$.)

In proving these results, a key step consists, for a fixed $D \in(0,1)$, in obtaining a necessary and sufficient condition for the Minkowski measurability of $\Gamma=\partial \Omega$ in terms of the sequence of lengths $\left(l_{j}\right)_{j=1}^{\infty}$ of the connected components (open intervals) of the open set $\Omega \subset \mathbb{R}$; namely, if $\left(l_{j}\right)_{j=1}^{\infty}$ is chosen to be non-increasing (which is always possible since $|\Omega|_{1}=\sum_{j=1}^{\infty} l_{j}<+\infty$ ), this condition is given by

$$
\lim _{j \rightarrow \infty} l_{j} j^{1 / D}=L, \quad \text { for some } L>0 .
$$

We also examine here the case when $\Gamma$ is not (necessarily) Minkowski measurable. In particular, in [16], the following conjecture was made (see [16, Conjecture 5.1', § 5.2, p. 521]):

CONJECTURe 1' (sharp remainder estimates). If $\Omega$ has 'fractal' boundary $\Gamma$ with Minkowski dimension $D \in(n-1, n)$ and if $0<\mathcal{M}_{*}(D ; \Gamma) \leqslant \mathcal{M}^{*}(D ; \Gamma)<+\infty$, then $\lambda^{-D / 2}(\varphi(\lambda)-N(\lambda))$ is bounded between two positive constants for all large $\lambda$.

In this paper, we not only prove Conjecture $1^{\prime}$ for $n=1$, we show the converse as well. (See Theorem 2.4.)

A good illustration of this result is provided by the Cantor set, studied in Example 4.5 below. Let $\Omega$ be the complement in $[0,1]$ of the triadic Cantor set $\Gamma$. Then $\Gamma=\partial \Omega$ and the sequence $\left(l_{j}\right)_{j=1}^{\infty}$ of interval lengths is given by

$$
\frac{1}{3}, \frac{1}{9}, \frac{1}{9}, \frac{1}{27}, \frac{1}{27}, \frac{1}{27}, \frac{1}{27}, \ldots,
$$

where there are $2^{k-1}$ copies of $3^{-k}$, for $k=1,2, \ldots$. Then clearly, (1.14) does not hold, so that $\Gamma$ is not Minkowski measurable. However, the hypothesis of Conjecture $1^{\prime}$ holds, so that from our result in the one-dimensional case, the two-sided estimate of Conjecture $1^{\prime}$ holds. In fact, we prove that the asymptotic expansion of $N(\lambda)$ does not admit a second term (or, more precisely, that $\lambda^{-D / 2}(\varphi(\lambda)-N(\lambda))$ does not converge, as $\left.\lambda \rightarrow+\infty\right)$.

It is somewhat a surprise to us that the supposedly 'trivial' case $n=1$ contains so many non-trivial results. The natural question is how much of this work generalizes to the cases where $n \geqslant 2$. In a subsequent paper, we shall show in fact that Conjecture 1 is not true when $n \geqslant 2$. It turns out that, at least for several classes of examples, what plays the role of $\zeta(s)$ in the case where $n=1$ are various Epstein zeta-functions and other Dirichlet series. We still conjecture that Minkowski measurability implies the existence of a second term in the expansion of $N(\lambda)$ that is proportional to $\lambda^{D / 2}$. However, the constant coefficient of this term must depend on $\Omega$ in a more complex way than is suggested in the conjecture. In fact, a revised form of Conjecture 1 will be presented in this forthcoming paper that gives the constant in terms of a limit involving the spectral zeta-function associated with the boundary value problem (P). 
The remainder of this paper is organized as follows. In the next section, we present more precisely some of our results. In $\S 3$, we prove, in particular, Conjecture $1^{\prime}$, as well as its converse, when $n=1$. We also give several examples that illustrate the pathologies that may occur (see $\S 3.3$ ). In $\S 4$, we prove the 'MWB conjecture' (Conjecture 1) when $n=1$ and establish in the process the connection with the Riemann zeta-function (see $\S 4.2$ ). We also obtain the aforementioned characterization of 'Minkowski measurability' (see §4.1). Moreover, in $\S 4.3$, we illustrate our results by two instructive examples.

Some of the results of this paper were announced earlier in [22].

\section{Statement of the main results}

Let $\Omega$ be a (non-empty) open subset of $\mathbb{R}$, of finite length $|\Omega|_{1}$ and with boundary $\Gamma=\partial \Omega$. We write $\Omega$ as the union of its connected components:

$$
\Omega=\bigcup_{j=1}^{\infty} I_{j}
$$

where the open intervals $I_{j}$ are pairwise disjoint and of length $l_{j}$. Since $|\Omega|_{1}=\sum_{j=1}^{\infty} l_{j}<+\infty$, we can assume without loss of generality that

$$
l_{1} \geqslant l_{2} \geqslant \ldots \geqslant l_{j} \geqslant \ldots>0 \text {. }
$$

(The case when $\Omega$ is a finite union of open intervals is of no interest here. Further, the 'lengths' $l_{j}$ are repeated according to their 'multiplicity'.)

Unless explicitly stated otherwise, $\left(l_{j}\right)_{j=1}^{\infty}$ will always denote the sequence associated with $\Omega$ in this manner.

Recall that the eigenvalues of the differential operator $-d^{2} / d y^{2}$ on the bounded open interval $I:=(\alpha, \beta)$ with Dirichlet boundary conditions at $\alpha$ and $\beta(u(\alpha)=u(\beta)=0)$, are $\mu_{k}=(\pi / l)^{2} k^{2}$, for $k=1,2, \ldots$, where $l=\beta-\alpha$. Let $N(\lambda ; I)$ denote the associated eigenvalue counting function. Then if $[v]$ denotes the integer part of $v$, it follows that for $\lambda>0$,

$$
N(\lambda ; I)=\#\{k \geqslant 1: k \leqslant l(\sqrt{ } \lambda) / \pi\}=[l(\sqrt{ } \lambda) / \pi] .
$$

Consequently, we deduce from (2.1) and, for example, [16, Lemma 4.2, p. 493], that

$$
N(\lambda)=\sum_{j=1}^{\infty} N\left(\lambda ; I_{j}\right)=\sum_{j=1}^{\infty}\left[l_{j} x\right], \quad \text { where } x:=\pi^{-1} \lambda^{\frac{1}{2}}
$$

(Recall that $N(\lambda)$ denotes the eigenvalue 'counting function' associated with $\Omega$.)

Let $\zeta(s)$ denote the Riemann zeta-function (see, for example, [32]). Recall that $\zeta(s)=\sum_{j=1}^{\infty} j^{-s}$ for $\operatorname{Re} s>1$ and that $\zeta(s)$ has a meromorphic extension to all of $\mathbb{C}$ with a single, simple pole (with residue 1) located at the point $s=1$. In particular, the meromorphic continuation of $\zeta$ to the region $\operatorname{Re} s>0$ is given by

$$
\zeta(s)=\frac{1}{s-1}+\int_{1}^{+\infty}\left([t]^{-s}-t^{-s}\right) d t, \quad \text { for } \operatorname{Re} s>0
$$

indeed, this identity is obvious for $\operatorname{Re} s>1$ and the integral in the right-hand side of (2.3) is analytic for $\operatorname{Re} s>0$. 


\subsection{Existence of a second term: resolution of Conjecture 1}

We can now state our first result:

THEOREM 2.1. (a) Let $\left(l_{j}\right)_{j=1}^{\infty}$ be an arbitrary, positive, non-increasing sequence such that

Then

$$
l_{j} \sim L j^{-1 / D}, \quad \text { as } j \rightarrow \infty, \text { for } D \in(0,1) \text { and } L>0 .
$$

$$
\sum_{j=1}^{\infty}\left[l_{j} x\right]=\left(\sum_{j=1}^{\infty} l_{j}\right) x+\zeta(D) L^{D} x^{D}+o\left(x^{D}\right), \quad \text { as } x \rightarrow+\infty .
$$

(b) In particular, if the sequence $\left(l_{j}\right)_{j=1}^{\infty}$ associated with $\Omega$ satisfies hypothesis (2.4) and if $\varphi(\lambda):=\pi^{-1}|\Omega|_{1} \lambda^{\frac{1}{2}}$, we deduce from (2.2) and (2.5) that

$$
N(\lambda)=\varphi(\lambda)+\pi^{-D} \zeta(D) L^{D} \lambda^{D / 2}+o\left(\lambda^{D / 2}\right), \quad \text { as } \lambda \rightarrow+\infty .
$$

Recall that in (2.4), the condition $l_{j} \sim L j^{-1 / D}$ means that $l_{j} j^{1 / D} \rightarrow L$, as $j \rightarrow \infty$.

The following theorem provides, in particular, a characterization (of independent interest) of the case when $\Gamma=\partial \Omega$ is Minkowski measurable with $D=$ $D(\Gamma) \in(0,1)$.

THEOREM 2.2 (characterization of Minkowski measurability). The sequence $\left(l_{j}\right)_{j=1}^{\infty}$ associated with $\Omega$ satisfies hypothesis (2.4) if and only if $\Gamma=\partial \Omega$ is Minkowski measurable and has Minkowski dimension $D \in(0,1)$ (that is, if and only if the hypotheses of the 'modified Weyl-Berry conjecture' are satisfied). In addition, in this case, we have

$$
M(D ; \Gamma)=\frac{2^{1-D}}{1-D} L^{D}
$$

By combining Theorems 2.1(b) and 2.2, we can now establish Conjecture 1 [16, Conjecture 5.1, p. 520] in the case when $n=1$. We also obtain the exact value of the constant $c_{1, D}$ which is expressed in terms of $\zeta(D)$.

Corollary 2.3 (resolution of the modified Weyl-Berry conjecture in the one-dimensional case). Let $\Omega$ be a bounded open subset of $\mathbb{R}$ such that $\Gamma=\partial \Omega$ is Minkowski measurable and of Minkowski dimension $D \in(0,1)$. Then

$$
N(\lambda)=\varphi(\lambda)-c_{1, D} M(D ; \Gamma) \lambda^{D / 2}+o\left(\lambda^{D / 2}\right), \quad \text { as } \lambda \rightarrow+\infty,
$$

where $\varphi(\lambda)=\pi^{-1}|\Omega|_{1} \lambda^{\frac{1}{2}}$ and the positive constant $c_{1, D}$ is given by

$$
c_{1, D}:=2^{-(1-D)} \pi^{-D}(1-D)(-\zeta(D)) .
$$

\subsection{Sharp remainder estimates: resolution of Conjecture $1^{\prime}$}

Let $\{v\}:=v-[v]$ in $[0,1)$ denote the fractional part of the real number $v$. If $f(x), g(x)$ are real-valued functions defined on a subset of $\mathbb{R}$, we write ' $f(x)=g(x)$ as $x \rightarrow a$ ' if there are positive constants $c_{1}, c_{2}$ such that $c_{1} f(x) \leqslant$ $g(x) \leqslant c_{2} f(x)$ for all $x$ in some neighbourhood of $a$ and in the domains of both $f$ and $g$. Here, $a \in \mathbb{R} \cup\{ \pm \infty\}$.

The following result not only settles Conjecture $1^{\prime}$ in the case where $n=1$, but the converse and more. 
THEOREM 2.4. Let $\Omega$ be a bounded open subset of $\mathbb{R}$, let $\left(l_{j}\right)_{j=1}^{\infty}$ be the associated sequence, and let. $D \in(0,1)$. The following assertions are equivalent:

(i) $l_{j}=j^{-1 / D}$ as $j \rightarrow \infty$;

(ii) $\Gamma=\partial \Omega$ has Minkowski dimension $D$ and

$$
0<\mathcal{M}_{*}(D ; \Gamma) \leqslant \mathcal{M}^{*}(D ; \Gamma)<+\infty ;
$$

(iii) $\sum_{j=1}^{\infty}\left\{l_{j} x\right\} \asymp x^{D}$ as $x \rightarrow+\infty$;

(iv) $\varphi(\lambda)-N(\lambda)=\lambda^{D / 2}$ as $\lambda \rightarrow+\infty$.

Note that (2.2) gives the identity $\varphi(\lambda)-N(\lambda)=\sum_{j=1}^{\infty}\left\{l_{j} x\right\}$ with $x=\pi^{-1} \lambda^{\frac{1}{2}}$, so that the equivalence (iii) $\Leftrightarrow$ (iv) in Theorem 2.4 is immediate.

We shall also provide additional results connected with the one-sided estimates of [16, Theorem 2.1 and Corollary 2.1, pp. 479-480] (see § 3.3). In particular, we shall show that when $n=1$ and $D \in(0,1)$, the error estimate (1.12) is equivalent to the hypothesis $\mathcal{M}^{*}(D ; \Gamma)<+\infty$. (See Theorem 3.10.)

Note that the assumption in our results that $\Omega$ is bounded may be relaxed to $|\Omega|_{1}<+\infty$.

Since, when $n=1$, the Sobolev space $H_{0}^{1}(\Omega) \subset H^{1}(\Omega)$ is composed of continuous functions (see, for example, [5, Theorem VIII.7, p. 129]), the Dirichlet boundary condition ' $u=0$ on $\Gamma$ ' in equation (1.1) can be understood pointwise here.

We can of course deduce (by an abelian argument) from the results of $\S 2.2$ two-sided estimates for the asymptotics of the 'partition function'

$$
Z(t)=\sum_{r=1}^{\infty} e^{-\lambda_{r} t}=\int_{0}^{+\infty} e^{-\lambda t} d N(\lambda) ;
$$

namely, (iv) of Theorem 2.4 yields

$$
\psi(t)-Z(t)=t^{-D / 2}, \quad \text { as } t \rightarrow 0^{+},
$$

where $\psi(t):=(4 \pi)^{-\frac{1}{2}}|\Omega|_{1} t^{-\frac{1}{2}}$. Similarly, we deduce from the results of $\S 2.1$ a second term for the asymptotics of $Z(t)$; namely, equation (2.8) yields

$$
Z(t)=\psi(t)-c_{1, D} \Gamma\left(1+\frac{1}{2} D\right) \mu(D ; \Gamma) t^{-D / 2}+o\left(t^{-D / 2}\right), \quad \text { as } t \rightarrow 0^{+},
$$

with $c_{1, D}$ given by (2.9). These results extend those of [6] in the case where $n=1$.

It is natural to wonder whether Theorem 2.1 admits a converse. After this work was completed, M. L. Lapidus and H. Maier [21] addressed this question and connected it with the Riemann hypothesis. More precisely, they have shown that a somewhat weaker form of the converse of Conjecture 1 when $n=1$ (equivalently, of Corollary 2.3 or of Theorem 2.1 above) is true for a given value of $D \in(0,1)$ if and only if $\zeta=\zeta(s)$ does not have a zero on the corresponding vertical line $\operatorname{Re} s=D$. In particular, it is not true when $D=\frac{1}{2}$ (the 'midfractal' case, in the sense of [16]), and is true everywhere else (that is, for all $D \in(0,1)$, $D \neq \frac{1}{2}$ ) if and only if the Riemann hypothesis is true.

\section{Proof of Conjecture 1' in the case of dimension one}

\subsection{Two-sided estimates}

We shall first derive expressions for $\mathcal{M}_{*}(D ; \Gamma)$ and $\mathcal{M}^{*}(D ; \Gamma)$ that depend only on the sequence $\left(l_{j}\right)_{j=1}^{\infty}$ associated with $\Omega$. Since $\Gamma_{\varepsilon} \cap \Omega=\bigcup_{j=1}^{\infty}\left(\left(\partial I_{j}\right)_{\varepsilon} \cap I_{j}\right)$, and 
since $\left|\left(\partial I_{j}\right)_{\varepsilon} \cap I_{j}\right|_{1}=\min \left(l_{j}, 2 \varepsilon\right)$, we have

where, for $\varepsilon>0$,

$$
\left|\Gamma_{\varepsilon} \cap \Omega\right|_{1}=\sum_{j=1}^{\infty}\left|\left(\partial I_{j}\right)_{\varepsilon} \cap I_{j}\right|_{1}=2 \varepsilon J(2 \varepsilon)+\sum_{j>J(2 \varepsilon)} l_{j},
$$

$$
J(\varepsilon):=\max \left\{j \geqslant 1: l_{j} \geqslant \varepsilon\right\} .
$$

(By convention, if $l_{j}<\varepsilon$ for every $j \geqslant 1$, we set $J(\varepsilon)=0$.) Hence, if

$$
L_{D}(\varepsilon):=2 \varepsilon^{D} J(2 \varepsilon)+\varepsilon^{D-1} \sum_{j>J(2 \varepsilon)} l_{j}
$$

we have that

$$
\mathcal{M}_{*}(D ; \Gamma)=\liminf _{\varepsilon \rightarrow 0^{+}} L_{D}(\varepsilon) \text { and } \mathcal{M}^{*}(D ; \Gamma)=\limsup _{\varepsilon \rightarrow 0^{+}} L_{D}(\varepsilon) .
$$

In the rest of this section, it will be convenient to adopt the following notation and terminology. If $D \in[0,1]$ and $\left(l_{j}\right)_{j=1}^{\infty}$ is any non-increasing sequence tending to zero, we set

$$
\mathcal{M}_{*}=\mathcal{M}_{*}\left(D ;\left(l_{j}\right)\right):=\liminf _{\varepsilon \rightarrow 0^{+}} L_{D}(\varepsilon), \quad \mathcal{M}^{*}=\mathcal{M}^{*}\left(D ;\left(l_{j}\right)\right):=\limsup _{\varepsilon \rightarrow 0^{+}} L_{D}(\varepsilon),
$$

where $L_{D}(\varepsilon)$ is defined by (3.2), with $J(\varepsilon)$ given by (3.1). We define the 'Minkowski dimension' of $\left(l_{j}\right)_{j=1}^{\infty}$ as the infimum of those $D$ with $\mu^{*}=0$. This is also equal to the supremum of those $D$ with $\mathcal{M}^{*}=+\infty$. If $0<\mu_{*}=\mu^{*}<+\infty$, we write $M=M\left(D ;\left(l_{j}\right)\right)$ for this common value. We then say that the sequence $\left(l_{j}\right)_{j=1}^{\infty}$ is 'Minkowski measurable' (with Minkowski dimension $D$ ) and has 'Minkowski content' $M$. (Of course, if $\left(l_{j}\right)_{j=1}^{\infty}$ is the sequence associated with the open set $\Omega$, then $\Gamma=\partial \Omega$ is Minkowski measurable if and only if $\left(l_{j}\right)_{j=1}^{\infty}$ is; moreover, in this case, by (3.3) and (3.4), the associated values of $\mathcal{M}$ coincide.)

Further, we let

$$
\alpha=\alpha\left(D ;\left(l_{j}\right)\right):=\liminf _{j \rightarrow \infty} l_{j} j^{1 / D} \text { and } \beta=\beta\left(D ;\left(l_{j}\right)\right):=\limsup _{j \rightarrow \infty} l_{j} j^{1 / D} .
$$

We can now state the following result that will be used, in particular, in the proofs of Theorems 2.4 and 2.2:

THEOREM 3.1. Given $D \in(0,1)$, let $\left(l_{j}\right)_{j=1}^{\infty}$ be any positive non-increasing sequence such that $0<\alpha \leqslant \beta<+\infty$ (that is, $l_{j}=j^{-1 / D}$, as $j \rightarrow \infty$ ).

(a) Then $\left(l_{j}\right)_{j=1}^{\infty}$ has Minkowski dimension $D$ and

$$
f(\alpha, \beta) \leqslant \mu_{*} \leqslant \mathcal{M}^{*} \leqslant f(\beta, \alpha),
$$

where

$$
f(u, v):=2^{1-D}\left(u^{D}+\frac{D}{1-D} u v^{D-1}\right) .
$$

(b) In particular, if $\alpha=\beta=L$, for some $L>0$ (that is, if $l_{j} \sim L j^{-1 / D}$, as $j \rightarrow \infty$ ), then $\left(l_{j}\right)_{j=1}^{\infty}$ is Minkowski measurable (with Minkowski dimension $D$ ) and its Minkowski content is given by

$$
M\left(D ;\left(l_{j}\right)\right)=\frac{2^{1-D}}{1-D} L^{D} .
$$


Proof. Part (b) clearly follows from Part (a). We now establish Part (a). Let $\alpha_{j}$ be defined by $l_{j}=\alpha_{j} j^{-1 / D}$. Thus, by (3.5), $\alpha=\lim \inf \alpha_{j}$ and $\beta=\lim \sup \alpha_{j}$. From the definition (3.1) of $J(\varepsilon)$, we have

Thus

$$
J(2 \varepsilon)=\max \left\{j \geqslant 1: j \leqslant(2 \varepsilon)^{-D}\left(\alpha_{j}\right)^{D}\right\} .
$$

$$
(\alpha+o(1))^{D}(2 \varepsilon)^{-D} \leqslant J(2 \varepsilon) \leqslant(\beta+o(1))^{D}(2 \varepsilon)^{-D}, \quad \text { as } \varepsilon \rightarrow 0^{+} .
$$

Note that since $D \in(0,1)$,

$$
\sum_{j>T} j^{-1 / D} \sim \frac{D}{1-D} T^{1-(1 / D)}, \quad \text { as } T \rightarrow+\infty .
$$

Thus

$$
\begin{aligned}
(\alpha+o(1)) \frac{D}{1-D}(J(2 \varepsilon))^{1-(1 / D)} & \leqslant \sum_{j>J(2 \varepsilon)} \alpha_{j} j^{-1 / D} \\
& \leqslant(\beta+o(1)) \frac{D}{1-D}(J(2 \varepsilon))^{1-(1 / D)},
\end{aligned}
$$

$$
\text { as } \varepsilon \rightarrow 0^{+} \text {. }
$$

Putting (3.9) into (3.10) and noting that $1-(1 / D)<0$, we have as $\varepsilon \rightarrow 0^{+}$,

$$
\begin{aligned}
(\alpha+o(1))(\beta+o(1))^{D-1}(2 \varepsilon)^{1-D} & \frac{D}{1-D} \\
& \leqslant \sum_{j>J(2 \varepsilon)} \alpha_{j} j^{-1 / D}=\sum_{j>J(2 \varepsilon)} l_{j} \\
& \leqslant(\beta+o(1))(\alpha+o(1))^{D-1}(2 \varepsilon)^{1-D} \frac{D}{1-D} .
\end{aligned}
$$

Multiplying (3.9) by $2 \varepsilon^{D}$ and (3.11) by $\varepsilon^{D-1}$, we deduce upon adding them that, as $\varepsilon \rightarrow 0^{+}$,

$$
\begin{aligned}
2^{1-D}\left(\alpha^{D}+\alpha \beta^{D-1} \frac{D}{1-D}+o(1)\right) & \leqslant L_{D}(\varepsilon)=2 \varepsilon^{D} J(2 \varepsilon)+\varepsilon^{D-1} \sum_{j>J(2 \varepsilon)} l_{j} \\
& \leqslant 2^{1-D}\left(\beta^{D}+\beta \alpha^{D-1} \frac{D}{1-D}+o(1)\right) .
\end{aligned}
$$

In view of definition (3.4), we now obtain (3.6) by taking the lower and upper limits as $\varepsilon \rightarrow 0^{+}$in (3.12). Further, (3.6) implies that $\left(l_{j}\right)_{j=1}^{\infty}$ has Minkowski dimension $D$. This establishes Part (a) and concludes the proof of Theorem 3.1.

Here and henceforth, it will be convenient to adopt the following notation. If $\left(l_{j}\right)_{j=1}^{\infty}$ is any positive non-increasing sequence such that $\sum_{j=1}^{\infty} l_{j}<+\infty$, we set, for $x>0$,

$$
\delta(x):=\sum_{j=1}^{\infty} l_{j} x-\sum_{j=1}^{\infty}\left[l_{j} x\right]=\sum_{j=1}^{\infty}\left\{l_{j} x\right\}
$$

moreover, we let

$$
\delta_{*}:=\liminf _{x \rightarrow+\infty} x^{-D} \delta(x) \text { and } \delta^{*}:=\limsup _{x \rightarrow+\infty} x^{-D} \delta(x) .
$$

The previous theorem contained the implication (i) $\Rightarrow$ (ii) in Theorem 2.4. The next theorem contains the implication (i) $\Rightarrow$ (iii). 
Theorem 3.2. Given $D \in(0,1)$, let $\left(l_{j}\right)_{j=1}^{\infty}$ be an arbitrary positive nonincreasing sequence such that $0<\alpha \leqslant \beta<+\infty$ (that is, $l_{j}=j^{-1 / D}$, as $j \rightarrow \infty$ ). Then

$$
\frac{D}{1-D} \alpha \beta^{D-1} \leqslant \delta_{*} \leqslant \delta^{*} \leqslant \beta^{D}+\frac{D}{1-D} \beta \alpha^{D-1} .
$$

Proof. We have

$$
\delta(x)=\sum_{j \leqslant J\left(x^{-1}\right)}\left\{l_{j} x\right\}+\sum_{j>J\left(x^{-1}\right)}\left\{l_{j} x\right\}=\sum_{j \leqslant J\left(x^{-1}\right)}\left\{l_{j} x\right\}+x \sum_{j>J\left(x^{-1}\right)} l_{j} ;
$$

so that using $0 \leqslant\{v\}<1$ for all $v$, we obtain

$$
x^{1-D} \sum_{j>J\left(x^{-1}\right)} l_{j} \leqslant x^{-D} \delta(x) \leqslant x^{-D} J\left(x^{-1}\right)+x^{1-D} \sum_{j>J\left(x^{-1}\right)} l_{j} .
$$

From (3.9) with $2 \varepsilon=x^{-1}$, we have as $x \rightarrow+\infty$,

$$
x^{-D} J\left(x^{-1}\right) \leqslant \beta^{D}+o(1) .
$$

Similarly, from (3.11) with $2 \varepsilon=x^{-1}$, we have as $x \rightarrow+\infty$,

$$
\alpha \beta^{D-1} \frac{D}{1-D}+o(1) \leqslant x^{1-D} \sum_{j>J\left(x^{-1}\right)} l_{j} \leqslant \beta \alpha^{D-1} \frac{D}{1-D}+o(1) .
$$

Putting these inequalities into (3.16) yields, as $x \rightarrow+\infty$,

$$
\alpha \beta^{D-1} \frac{D}{1-D}+o(1) \leqslant x^{-D} \delta(x) \leqslant \beta^{D}+\beta \alpha^{D-1} \frac{D}{1-D}+o(1) ;
$$

from which, in view of definition (3.14), the conclusion (3.15) of Theorem 3.2 follows at once.

\subsection{Proof of Conjecture 1' and of its converse}

The following result contains the implication (iii) $\Rightarrow$ (i) in Theorem 2.4.

Theorem 3.3. Let $l_{1} \geqslant l_{2} \geqslant \ldots>0$ be such that $\sum_{j=1}^{\infty} l_{j}<+\infty$ and let $\delta(x)$ be defined by (3.13). Assume that for some $D \in(0,1)$,

$$
\delta(x) \asymp x^{D} \text {, as } x \rightarrow+\infty .
$$

Then

$$
l_{j}=j^{-1 / D}, \quad \text { as } j \rightarrow \infty .
$$

Proof. By assumption, there exist positive constants, $c_{1}, c_{2}$ and $x_{0}$ such that

$$
c_{1} x^{D} \leqslant \delta(x) \leqslant c_{2} x^{D}, \text { for all } x \geqslant x_{0} .
$$

Let $k \geqslant 2$ be a fixed integer with $k>\left(2 c_{2} / c_{1}\right)^{1 /(1-D)}$, so that

$$
c_{2} k^{D}<\frac{1}{2} c_{1} k \text {. }
$$

Let

$$
U:=\sum_{\left\{l_{j} x\right\}<k^{-1}}\left\{l_{j} x\right\} \text { and } V:=\sum_{\left\{l_{j} x\right\} \geqslant k^{-1}}\left\{l_{j} x\right\}
$$


so that

$$
\delta(x)=\sum_{j=1}^{\infty}\left\{l_{j} x\right\}=U+V .
$$

Thus, since $k\{v\}=\{k v\}$ for $\{v\}<k^{-1}$, it follows from (3.19) and (3.20) that for $x \geqslant x_{0}$ (and hence $k x \geqslant x_{0}$ ),

$$
\begin{aligned}
k U & =\sum_{\left\{l_{j} x\right\}<k^{-1}}\left\{l_{j} k x\right\} \leqslant \delta(k x) \\
& \leqslant c_{2} k^{D} x^{D} \leqslant \frac{1}{2} c_{1} k x^{D}<\frac{1}{2} k \delta(x) .
\end{aligned}
$$

Consequently, $U<\frac{1}{2} \delta(x)$ and hence, by (3.21b), $V>\frac{1}{2} \delta(x)$. We deduce from the last inequality that the number of $j$ with $\left\{l_{j} x\right\} \geqslant k^{-1}$ is greater than $\frac{1}{2} \delta(x)$ (since each $\left\{l_{j} x\right\}$ is less than 1 ); a fortiori, the same is true of the number of $j$ with $l_{j} x \geqslant k^{-1}$ or, equivalently, $l_{j} \geqslant(k x)^{-1}$.

Thus, with $J(\varepsilon)$ defined as in (3.1), it follows from the above discussion that

$$
J(1 / k x)>\frac{1}{2} \delta(x) \geqslant \frac{1}{2} c_{1} x^{D} .
$$

Since $l_{j} \downarrow 0$, there exists a positive integer $j_{1}$ such that $l_{j_{1}}<\left(k x_{0}\right)^{-1}$; so that $x_{0}<\left(k l_{j}\right)^{-1}$, for all $j \geqslant j_{1}$. Fix $j \geqslant j_{1}$ and consider $x$ with $x_{0} \leqslant x<\left(k l_{j}\right)^{-1}$; so that $l_{j}<(k x)^{-1}$. Then, by definition of $J(\varepsilon)$ and by (3.22),

$$
\frac{1}{2} c_{1} x^{D}<J(1 / k x)<j \text {. }
$$

This is true for all $x \in\left[x_{0}, 1 / k l_{j}\right)$; so letting $x \rightarrow\left(1 / k l_{j}\right)^{-}$in (3.23), we obtain $\frac{1}{2} c_{1}\left(1 / k l_{j}\right)^{D} \leqslant j$, which implies that

$$
l_{j} j^{1 / D} \geqslant k^{-1}\left(\frac{1}{2} c_{1}\right)^{1 / D} \quad \text { for all } j \geqslant j_{1} \text {. }
$$

Thus

$$
\alpha=\liminf _{j \rightarrow \infty} l_{j} j^{1 / D} \geqslant k^{-1}\left(\frac{1}{2} c_{1}\right)^{1 / D}>0 .
$$

Next, let $\sigma$ denote the number of $j \in(J(2 / x), J(1 / x)]$ with $\left\{l_{j} x\right\} \geqslant \frac{1}{2}$ and let $\tau$ denote the number of $j$ in this interval with $\left\{l_{j} x\right\}<\frac{1}{2}$. Then

$$
\sigma+\tau=J(1 / x)-J(2 / x) .
$$

But, by definition of $\sigma$ and $\delta(x)$,

$$
\frac{1}{2} \sigma \leqslant \delta(x) \text {. }
$$

Further, if $j$ is counted by $\tau$, then $\left\{l_{j} \frac{1}{2} x\right\} \geqslant \frac{1}{2}$. (Indeed, $J(1 / x) \geqslant j>J(2 / x)$ implies that $\frac{1}{2} \leqslant \frac{1}{2} l_{j} x<1$ and hence $\left\{l_{j} \frac{1}{2} x\right\}=\frac{1}{2} l_{j} x \geqslant \frac{1}{2}$.) So

$$
\frac{1}{2} \tau \leqslant \delta\left(\frac{1}{2} x\right) \text {. }
$$

Therefore, (3.25) yields

$$
\begin{aligned}
J(1 / x)-J(2 / x)=\sigma+\tau & \leqslant 2 \delta(x)+2 \delta\left(\frac{1}{2} x\right) \\
& \leqslant 2\left(1+2^{-D}\right) c_{2} x^{D} \leqslant 4 c_{2} x^{D}
\end{aligned}
$$

this is valid for all $x \geqslant 2 x_{0}$.

Now, for any $x \geqslant 2 x_{0}$, let $m=m(x)$ be that integer such that

$$
\frac{x}{2^{m}} \geqslant x_{0}>\frac{x}{2^{m+1}} \text {. }
$$


Then, since $x / 2^{m-1} \geqslant 2 x_{0}$, we can apply estimate (3.26) with $x$ replaced by $x / 2^{k}$, for $k=0, \ldots, m-1$, and since also $1 / 2 x_{0}<2^{m} / x$, we have successively

$$
\begin{aligned}
J\left(\frac{1}{x}\right) & =\sum_{k=0}^{m-1}\left(J\left(\frac{2^{k}}{x}\right)-J\left(\frac{2^{k+1}}{x}\right)\right)+J\left(\frac{2^{m}}{x}\right) \\
& \leqslant \sum_{k=0}^{m-1} 4 c_{2}\left(\frac{x}{2^{k}}\right)^{D}+J\left(\frac{1}{2 x_{0}}\right) \\
& \leqslant \sum_{k=0}^{\infty} 4 c_{2}\left(\frac{x}{2^{k}}\right)^{D}+J\left(\frac{1}{2 x_{0}}\right) \\
& =\frac{4 c^{2}}{1-2^{-D}} x^{D}+J\left(\frac{1}{2 x_{0}}\right) .
\end{aligned}
$$

Let $J_{0}:=J\left(1 / 2 x_{0}\right)$ and let $j>J_{0}$; further, let $x:=1 / l_{j}$, so that $x>2 x_{0}$. Then we deduce from (3.27) and (3.1) that

$$
J_{0}<j \leqslant J\left(\frac{1}{x}\right) \leqslant \frac{4 c_{2}}{1-2^{-D}} x^{D}+J_{0}=\frac{4 c_{2}}{1-2^{-D}}\left(l_{j}\right)^{-D}+J_{0},
$$

from which we obtain

$$
l_{j} j^{1 / D} \leqslant\left(\frac{j}{j-J_{0}}\right)^{1 / D}\left(\frac{4 c_{2}}{1-2^{-D}}\right)^{1 / D}
$$

Thus

$$
\beta=\limsup _{j \rightarrow \infty} l_{j} j^{1 / D} \leqslant\left(\frac{4 c_{2}}{1-2^{-D}}\right)^{1 / D}<+\infty .
$$

Together with (3.24), this yields (3.18) and hence proves Theorem 3.3.

REMARK 3.4. We have used the full hypothesis (3.17) to prove (3.24), but only the assumption $\delta^{*}:=\lim \sup _{x \rightarrow+\infty} x^{-D} \delta(x)<+\infty$ to prove (3.28). In other words, with the notation of (3.5) and (3.14), $\delta^{*}<+\infty$ implies that $\beta<+\infty$. On the other hand, $\alpha>0$ seems to require both $\delta_{*}>0$ and $\delta^{*}<+\infty$. We shall discuss this issue later in this section.

The next result contains part of the implication (ii) $\Rightarrow$ (i) in Theorem 2.4:

THEOREM 3.5. Suppose that $l_{1} \geqslant l_{2} \geqslant \ldots>0$ and $0<\mathcal{M}_{*} \leqslant \mathcal{M}^{*}<+\infty$, for some $D \in(0,1)$. Then $\alpha>0$.

We note that in the above statement, $\alpha>0$ means that $\alpha \in(0,+\infty]$. It should be clear from the context when we use such a convention elsewhere in the paper.

In order to prove Theorem 3.5, we shall use the following lemma.

LEMma 3.6. If $\lim \inf _{\varepsilon \rightarrow 0^{+}} \varepsilon^{D} J(\varepsilon)>0$, then $\alpha>0$.

Proof of Lemma 3.6. Let $\alpha_{j}:=l_{j} j^{1 / D}$. If $l_{j}=l_{j+1}$, then $\alpha_{j}<\alpha_{j+1}$. Thus

$$
\alpha=\liminf _{j \rightarrow \infty} \alpha_{j}=\liminf _{j: l_{j}>l_{j+1}} \alpha_{j+1} \text {. }
$$

Suppose $\lim _{\inf _{\varepsilon \rightarrow 0^{+}}} \varepsilon^{D} J(\varepsilon)>0$. Then there is some $C>0$ with $\varepsilon^{D} J(\varepsilon) \geqslant C$ for all $\varepsilon>0$. Say $l_{j}>l_{j+1}$. For any $\varepsilon$ in $\left(l_{j+1}, l_{j}\right)$, we have $J(\varepsilon)=j$ and hence $\varepsilon^{D_{j}} \geqslant C$; 
that is, $\varepsilon \geqslant C^{1 / D} j^{-1 / D}$. Since this is true for any $\varepsilon \in\left(l_{j+1}, l_{j}\right)$, we may let $\varepsilon \rightarrow l_{j+1}^{+}$ and obtain

$$
l_{j+1} \geqslant C^{1 / D_{j}^{-1 / D}}>C^{1 / D}(j+1)^{-1 / D} .
$$

Thus $\alpha_{j+1}=l_{j+1}(j+1)^{1 / D}>C^{1 / D}$ and hence

$$
\liminf _{j: l_{j}>l_{j+1}} \alpha_{j+1} \geqslant C^{1 / D}>0
$$

which in view of (3.29), implies that $\alpha>0$, as required.

Proof of Theorem 3.5. Since $\mathcal{M}^{*}<+\infty$, it follows from definitions (3.2) and (3.4) that there is some finite constant $M$ such that

$$
\varepsilon^{D-1} \sum_{j>J(2 \varepsilon)} l_{j} \leqslant M
$$

for all $\varepsilon>0$. From Lemma 3.6, in order to prove that $\alpha>0$, it is sufficient to show that $\liminf _{\varepsilon \rightarrow 0^{+}} \varepsilon^{D} J(\varepsilon)>0$. Suppose the contrary is true, that is, $\lim \inf _{\varepsilon \rightarrow 0^{+}} \varepsilon^{D} J(\varepsilon)=0$. Let $\varepsilon_{1}>\varepsilon_{2}>\ldots$ be a sequence such that $\lim _{q \rightarrow \infty}\left(\varepsilon_{q}\right)^{D} J\left(\varepsilon_{q}\right)$ $=0$. Let

$$
\theta_{q}:=\left(\varepsilon_{q}\right)^{D} J\left(\varepsilon_{q}\right)
$$

We may assume that each $\theta_{q}<1$. Let

$$
\eta_{q}:=\left(\theta_{q}\right)^{-\frac{1}{2}} \varepsilon_{q},
$$

so that $2 \eta_{q}>2 \varepsilon_{q}$ and hence $J\left(2 \eta_{q}\right) \leqslant J\left(2 \varepsilon_{q}\right) \leqslant J\left(\varepsilon_{q}\right)$. We then have, by (3.2),

$$
\begin{aligned}
L_{D}\left(\eta_{q}\right) & =\left(\eta_{q}\right)^{D-1} \sum_{j>J\left(2 \eta_{q}\right)} l_{j}+2\left(\eta_{q}\right)^{D} J\left(2 \eta_{q}\right) \\
& =\left(\eta_{q}\right)^{D-1} \sum_{J\left(2 \eta_{q}\right)<j \leq J\left(2 \varepsilon_{q}\right)} l_{j}+\left(\eta_{q}\right)^{D-1} \sum_{j>J\left(2 \varepsilon_{q}\right)} l_{j}+2\left(\eta_{q}\right)^{D} J\left(2 \eta_{q}\right) \\
& =: E_{q}+F_{q}+G_{q}, \text { say. }
\end{aligned}
$$

Now, in view of (3.31),

$$
\begin{aligned}
G_{q}=2\left(\eta_{q}\right)^{D} J\left(2 \eta_{q}\right) & \leqslant 2\left(\eta_{q}\right)^{D} J\left(\varepsilon_{q}\right) \\
& =2\left(\theta_{q}\right)^{-D / 2}\left(\varepsilon_{q}\right)^{D} J\left(\varepsilon_{q}\right) \\
& =2\left(\theta_{q}\right)^{1-(D / 2)} \leqslant 2\left(\theta_{q}\right)^{(1-D) / 2} .
\end{aligned}
$$

Similarly, since $l_{j}<2 \eta_{q}$ whenever $j>J\left(2 \eta_{q}\right)$, we have as in (3.33),

$$
\begin{aligned}
E_{q}=\left(\eta_{q}\right)^{D-1} \sum_{J\left(2 \eta_{q}\right)<j \leqslant J\left(2 \varepsilon_{q}\right)} l_{j} & \leqslant\left(\eta_{q}\right)^{D-1} 2 \eta_{q} J\left(2 \varepsilon_{q}\right) \\
& \leqslant 2\left(\eta_{q}\right)^{D J} J\left(\varepsilon_{q}\right) \\
& \leqslant 2\left(\theta_{q}\right)^{(1-D) / 2} .
\end{aligned}
$$

Finally,

$$
\begin{aligned}
F_{q}=\left(\eta_{q}\right)^{D-1} \sum_{j>J\left(2 \varepsilon_{q}\right)} l_{j} & =\left(\theta_{q}\right)^{(1-D) / 2}\left(\varepsilon_{q}\right)^{D-1} \sum_{j>J\left(2 \varepsilon_{q}\right)} l_{j} \\
& \leqslant M\left(\theta_{q}\right)^{(1-D) / 2},
\end{aligned}
$$

where we have used (3.30) in the last inequality of (3.35). 
Putting together (3.32)-(3.35), we obtain

$$
0<L_{D}\left(\eta_{q}\right) \leqslant(M+4)\left(\theta_{q}\right)^{(1-D) / 2} .
$$

But $\theta_{q}=\left(\varepsilon_{q}\right)^{D} J\left(\varepsilon_{q}\right) \rightarrow 0$, as $q \rightarrow \infty$; so $L_{D}\left(\eta_{q}\right) \rightarrow 0$, which contradicts the assumption $\mu_{*}>0$.

The following result is really a corollary of Remark 3.4 and of the proof of Theorem 3.2. It completes our proof of Theorem 2.4.

THeOREM 3.7. Let $l_{1} \geqslant l_{2} \geqslant \ldots>0$ with $\sum_{j=1}^{\infty} l_{j}<+\infty$. If $\mathcal{M}^{*}<+\infty$, then $\beta<+\infty$.

Proof. Recalling the definition (3.2) of $L_{D}(\varepsilon)$, we deduce from the easy inequality (3.16) that

$$
x^{-D} \delta(x) \leqslant x^{-D} J\left(x^{-1}\right)+x^{1-D} \sum_{j>J\left(x^{-1}\right)} l_{j}=2^{D-1} L_{D}\left(\frac{1}{2 x}\right) .
$$

Hence

$$
\delta^{*}=\limsup _{x \rightarrow+\infty} x^{-D} \delta(x) \leqslant \limsup _{x \rightarrow+\infty} 2^{D-1} L_{D}\left(\frac{1}{2 x}\right)=2^{D-1} \mathcal{M}^{*} .
$$

Since by assumption, $\mathcal{M}^{*}<+\infty$, we thus have $\delta^{*}<+\infty$. The fact that $\beta<+\infty$ now follows from Remark 3.4.

Remark 3.8. From the above proof, we deduce that $\mathcal{M}^{*}<+\infty$ implies $\delta^{*}<+\infty$, a (one-dimensional) result contained in [16].

\subsection{Further related results: one-sided estimates and examples}

Recall the definitions of $\mathcal{M}_{*}, \mathcal{M}^{*}, \alpha, \beta, \delta_{*}, \delta^{*}$ from (3.4), (3.5) and (3.14). These quantities are functions of a number $D \in[0,1]$ and a non-increasing sequence $\left(l_{j}\right)_{j=1}^{\infty}$ of positive numbers.

We establish here further results related either to Conjecture $1^{\prime}$ or to its converse. We obtain, in particular, an analogue of Theorem 2.4 for one-sided estimates; to do so, we shall need the following converse to Theorem 3.7.

THEOREM 3.9. Let $D \in(0,1)$. Assume that $l_{1} \geqslant l_{2} \geqslant \ldots>0$ and $\sum_{j=1}^{\infty} l_{j}<+\infty$. Then $\beta<+\infty$ implies that $\mathcal{M}^{*}<+\infty$.

Proof. Assume that $\beta<+\infty$. Then, in view of definitions (3.4) and (3.2), we have

$$
\mathcal{M}^{*}=\limsup _{\varepsilon \rightarrow 0^{+}}\left(2 \varepsilon^{D} J(2 \varepsilon)+\varepsilon^{D-1} \sum_{j>J(2 \varepsilon)} l_{j}\right) .
$$

It follows from (3.9) that

$$
2 \varepsilon^{D} J(2 \varepsilon) \leqslant 2^{1-D} \beta^{D}+o(1), \quad \text { as } \varepsilon \rightarrow 0^{+} ;
$$

thus there is some finite number $Q$ with

$$
J(\varepsilon) \leqslant \varepsilon^{-D} Q, \quad \text { for all } \varepsilon>0 .
$$


Now, by $(3.40)$ and since $J(2 \varepsilon) \leqslant J(\varepsilon) \leqslant J\left(\frac{1}{2} \varepsilon\right) \leqslant \ldots$ and $l_{j}<2^{-k+1} \varepsilon$ for $j>$ $J\left(2^{-k+1} \varepsilon\right)$,

$$
\begin{aligned}
\sum_{j>J(2 \varepsilon)} l_{j} & =\sum_{k=0}^{\infty} \sum_{j=J\left(2^{-k+1} \varepsilon\right)+1}^{J\left(2^{-k} \varepsilon\right)} l_{j} \\
& <\sum_{k=0}^{\infty} J\left(2^{-k} \varepsilon\right) 2^{-k+1} \varepsilon \leqslant \sum_{k=0}^{\infty}\left(2^{-k} \varepsilon\right)^{-D} Q 2^{-k+1} \varepsilon
\end{aligned}
$$

Thus, since $D-1<0$,

$$
\varepsilon^{D-1} \sum_{j>J(2 \varepsilon)} l_{j} \leqslant 2 Q \sum_{k=0}^{\infty}\left(2^{D-1}\right)^{k}=2 Q \frac{1}{1-2^{D-1}}<+\infty .
$$

In view of (3.38), our result follows from this inequality and (3.39).

We can now state the counterpart of Theorem 2.4 for one-sided (upper) estimates. It shows, in particular, that (when $n=1$ and $D \in(0,1)$ ) the conclusion of [16, Theorem 2.1, p. 479] (in the case of the Laplacian) is equivalent to its hypothesis.

THEOREM 3.10. Let $D \in(0,1)$ and let $\left(l_{j}\right)_{j=1}^{\infty}$ be an arbitrary non-increasing positive sequence such that $\sum_{j=1}^{\infty} l_{j}<+\infty$. Then the following statements are equivalent:

(i) $l_{j}=O\left(j^{-1 / D}\right)$, as $j \rightarrow \infty$;

(ii) $M^{*}<+\infty$;

(iii) $\delta(x)=O\left(x^{D}\right)$, as $x \rightarrow+\infty$;

and if, in addition, $\left(l_{j}\right)_{j=1}^{\infty}$ is the sequence associated with $\Omega$,

(iv) $N(\lambda)=\varphi(\ddot{i})+O\left(\lambda^{D / 2}\right)$, as $\lambda \rightarrow+\infty$.

Proof. The fact that (i) implies (ii) follows from Theorem 3.9; (ii) implies (iii) is just the one-dimensional case of [16, Theorem 2.1 or Corollary 2.1, pp. 479-480], and an independent proof of it was given above (see Remark 3.8). Finally, the fact that (iii) implies (i) follows from Remark 3.4. (Statements (iii) and (iv) are obviously equivalent.)

We now turn our attention to one-sided lower estimates. It turns out that the natural analogue to Theorem 3.10 is not true. First we record two simple implications concerning lower estimates.

TheOREM 3.11. Let $D \in(0,1)$ and let $\left(l_{j}\right)_{j=1}^{\infty}$ be an arbitrary non-increasing positive sequence such that $\sum_{j=1}^{\infty} l_{j}<+\infty$. Then $\alpha>0$ implies $\mu_{*}>0$ and $\delta_{*}>0$ implies $\mu_{*}>0$.

Proof. In view of definitions (3.2) and (3.4), the first assertion follows immediately from the first inequality in (3.9). The second assertion follows immediately from taking $x \rightarrow+\infty$ in (3.37).

It turns out that there are no other relationships between $\alpha, \mu_{*}$ and $\delta_{*}$ than those given in Theorem 3.11. That is, it is possible for $\alpha>0$ with $\delta_{*}=0$, for $\mu_{*}>0$ with $\alpha=\delta_{*}=0$, and for $\delta_{*}>0$ with $\alpha=0$. The following examples illustrate these phenomena. 
EXAMPLE 3.12. Consider the multi-set of elements $l_{j}$ where we have $2^{(k+1)^{2}}$ copies of $5^{-k^{2}}$ for $k=1,2, \ldots$. Then $D=\log 2 / \log 5, \alpha=1>0$ and $\delta_{*}=0$. The proof is routine and left to the reader (as with the next two examples). The authors would be happy to send details upon request.

EXAMPLE 3.13. Consider the multi-set of $l_{j}$ where we have $\left[2^{2^{k+1} / 3}\right]$ copies of $4^{-2^{k-1}}$, for $k=1,2, \ldots$. Then $D=\frac{1}{2}, \alpha=\delta_{*}=0$ and $\mu_{*}=2^{\frac{3}{2}}>0$.

EXAMPLE 3.14. Consider the set of $l_{j}$ where we have $\left[2^{2^{k+1 / 3}}\right]$ numbers in arithmetic progression from $4^{-2^{k-1}}$ to $4^{-2^{k+1 / 3}}$, for $k=1,2, \ldots$. Then $D=\frac{1}{2}, \alpha=0$ and $0<\delta_{*}<+\infty$.

\section{Proof of Conjecture 1 in the case of dimension one}

\subsection{Characterization of Minkowski measurability}

Let $\left(l_{j}\right)_{j=1}^{\infty}$ be a non-increasing sequence of positive numbers such that $\sum_{j=1}^{\infty} l_{j}<+\infty$ and let $L_{D}(\varepsilon)$ be given by $(3.2)$, with $D \in(0,1)$. We have shown in Theorem 3.1(b) that if $l_{j} \sim L j^{-1 / D}$, for some $L>0$, then the sequence $\left(l_{j}\right)_{j=1}^{\infty}$ is Minkowski measurable (that is, $\lim _{\varepsilon \rightarrow 0^{+}} L_{D}(\varepsilon)$ exists in $(0,+\infty)$ ) with Minkowski dimension $D$ and its Minkowski content $\mathcal{M}$ is given by

$$
\mu:=\lim _{\varepsilon \rightarrow 0^{+}} L_{D}(\varepsilon)=\frac{2^{1-D}}{1-D} L^{D} .
$$

We establish here the converse of this result and hence characterize the situation when $\left(l_{j}\right)_{j=1}^{\infty}$ is Minkowski measurable with $D \in(0,1)$. Hence Theorem 2.2 follows from Theorem 3.1(b) and Theorem 4.1 below.

THEOREM 4.1. Suppose that $\left(l_{j}\right)_{j=1}^{\infty}$ is a non-increasing sequence of positive numbers with $\sum_{j=1}^{\infty} l_{j}<+\infty$ and such that for some $L>0$ and $D \in(0,1)$, the following limit exists and is given by

$$
\lim _{\varepsilon \rightarrow 0^{+}} L_{D}(\varepsilon)=2^{1-D} \frac{L^{D}}{1-D}
$$

Then we have

$$
l_{j} \sim L j^{-1 / D}, \quad \text { as } j \rightarrow \infty .
$$

Proof. The proof of Theorem 4.1 is rather long and difficult, although of an elementary nature; it is divided into two steps. The heart of the proof, provided in Step 1, consists in establishing the theorem under the assumption that the sequence $\left(l_{j}\right)_{j=1}^{\infty}$ is strictly monotone and $l_{j+1} / l_{j} \rightarrow 1$. Then, in Step 2 , we show that the general case can always be reduced to the special case treated in Step 1.

Step 1 . We assume that $\left(l_{j}\right)_{j=1}^{\infty}$ is strictly decreasing and that $l_{j+1} / l_{j} \rightarrow 1$, as $j \rightarrow \infty$.

For each $j$, define $\alpha_{j}, \beta_{j}$ by the equations

$$
l_{j}=\alpha_{j} j^{-1 / D}, \quad \sum_{k>j} l_{k}=\beta_{j} \frac{D}{1-D} j^{1-(1 / D)} .
$$


Since $\left(l_{j}\right)_{j=1}^{\infty}$ is strictly decreasing, we have, with $J(\varepsilon)$ defined by (3.1),

$$
J\left(l_{j}\right)=j, \quad \text { for all } j \geqslant 1 .
$$

Then, in view of (3.2),

$$
\begin{aligned}
L_{D}\left(\frac{1}{2} l_{j}\right) & =2^{1-D}\left(l_{j}\right)^{D} j+2^{1-D}\left(l_{j}\right)^{D-1} \sum_{k>j} l_{k} \\
& =2^{1-D}\left(\alpha_{j}\right)^{D}+2^{1-D}\left(\alpha_{j}\right)^{D-1} \beta_{j} \frac{D}{1-D} .
\end{aligned}
$$

Since $l_{j} \rightarrow 0^{+}$, it thus follows from (4.1) that

$$
\lim _{j \rightarrow \infty}\left(\left(\alpha_{j}\right)^{D}+\left(\alpha_{j}\right)^{D-1} \beta_{j} \frac{D}{1-D}\right)=\frac{L^{D}}{1-D} .
$$

We now show that

$$
\limsup _{j \rightarrow \infty} \beta_{j} \leqslant L .
$$

Define a positive number $\gamma_{j}$ by the equation

$$
\left(\alpha_{j}\right)^{D}+\left(\alpha_{j}\right)^{D-1} \beta_{j} \frac{D}{1-D}=\frac{\left(\gamma_{j}\right)^{D}}{1-D}
$$

From (4.3), $\gamma_{j} \rightarrow L$, as $j \rightarrow \infty$. For fixed $b>0$ and $D \in(0,1)$, the minimum value of $a^{D}+\left(a^{D-1} b D /(1-D)\right)$ on the interval $(0,+\infty)$ occurs when $a=b$ and is equal to $b^{D} /(1-D)$. Thus $\left(\beta_{j}\right)^{D} /(1-D) \leqslant\left(\gamma_{j}\right)^{D} /(1-D)$ and hence $\beta_{j} \leqslant \gamma_{j}$ for each $j$, which proves (4.4).

Next we show that

$$
\liminf _{j \rightarrow \infty} \alpha_{j} \leqslant L \text { and } \quad \limsup _{j \rightarrow \infty} \alpha_{j} \geqslant L .
$$

If the first inequality in (4.5) fails, then there is some $\theta>0$ with $\alpha_{j}>L+\theta$, for all large $j$. Then for large $j$,

$$
\begin{aligned}
\beta_{j} \frac{D}{1-D} j^{1-(1 / D)}=\sum_{k>j} l_{k} & =\sum_{k>j} \alpha_{k} k^{-1 / D}>(L+\theta) \sum_{k>j} k^{-1 / D} \\
& >(L+\theta) \int_{j+1}^{+\infty} t^{-1 / D} d t=(L+\theta) \frac{D}{1-D}(j+1)^{1-(1 / D)},
\end{aligned}
$$

where the last inequality holds since $D \in(0,1)$ and the function $t^{-1 / D}$ is decreasing for $t>0$. Thus lim inf $\beta_{j} \geqslant L+\theta$, which contradicts (4.4).

To show the second inequality in (4.5), let

$$
\rho:=\limsup _{j \rightarrow \infty} \alpha_{j}
$$

Then an argument analogous to the one just completed shows that

$$
\limsup _{j \rightarrow \infty} \beta_{j} \leqslant \limsup _{j \rightarrow \infty} \alpha_{j}=\rho \text {. }
$$


Let $j_{1}<j_{2}<\ldots$ be a sequence of natural numbers for which $\lim _{k \rightarrow \infty} \alpha_{j_{k}}=\rho$. Then $\beta_{j_{k}} \leqslant \alpha_{j_{k}}+o(1)$, as $k \rightarrow \infty$. Thus

$$
\begin{aligned}
\frac{\left(\gamma_{j_{k}}\right)^{D}}{1-D}=\left(\alpha_{j_{k}}\right)^{D}+\left(\alpha_{j_{k}}\right)^{D-1} \beta_{j_{k}} \frac{D}{1-D} & \leqslant\left(\alpha_{j_{k}}\right)^{D}+\left(\alpha_{j_{k}}\right)^{D} \frac{D}{1-D}+o\left(\left(\alpha_{j_{k}}\right)^{D-1}\right) \\
& =\frac{\left(\alpha_{j_{k}}\right)^{D}}{1-D}+o\left(\left(\alpha_{j_{k}}\right)^{D-1}\right), \quad \text { as } k \rightarrow \infty .
\end{aligned}
$$

By Theorem 3.5, we have $\rho>0$. (Note that $\rho$ was denoted by $\beta$ in $\S 3$.) So that $o\left(\left(\alpha_{j_{k}}\right)^{D-1}\right)=o(1)$, as $k \rightarrow \infty$. Since $\gamma_{j} \rightarrow L$ and $\alpha_{j_{k}} \rightarrow \rho$, we deduce that

$$
\frac{L^{D}}{1-D} \leqslant \frac{\rho^{D}}{1-D}
$$

showing that $L \leqslant \rho$ and establishing (4.5).

Next, suppose $\lim \sup _{j \rightarrow \infty} \alpha_{j}>L$. Then there are some $\theta>0$ and an infinite set of subscripts $j$ with $\alpha_{j}>L+\theta$. Let

$$
r_{\theta}=\left(\frac{L+\frac{1}{2} \theta}{L+\theta}\right)^{D}
$$

so that $0<r_{\theta}<1$. If $j_{1}$ is large with $\alpha_{j_{1}}>L+\theta$, let $j_{0}=\left[r_{\theta} j_{1}\right]$ and let $j_{2}$ be the least subscript greater than $j_{1}$ with $\alpha_{j_{2}}<L+\left(1 / j_{1}\right)$. By the first inequality in (4.5), $j_{2}$ exists. Since $l_{j+1} / l_{j} \rightarrow 1$, by assumption, we have $\alpha_{j+1} / \alpha_{j} \rightarrow 1$, which implies that $\alpha_{j_{2}} \rightarrow L$ as $j_{1} \rightarrow \infty$ through those numbers with $\alpha_{j_{1}}>L+\theta$. Thus by (4.3), $\beta_{j_{2}} \rightarrow L$.

If $j_{0}<j<j_{1}$, then $l_{j}>l_{j_{1}}$ so that

$$
\alpha_{j}>\alpha_{j_{1}}\left(\frac{j}{j_{1}}\right)^{1 / D}>\alpha_{j_{1}}\left(r_{\theta}\right)^{1 / D}=\alpha_{j_{1}} \frac{L+\frac{1}{2} \theta}{L+\theta}>L+\frac{1}{2} \theta .
$$

Further, if $j_{1}<j<j_{2}$, then $\alpha_{j}>L$. Hence

$$
\begin{aligned}
\beta_{j_{0}} \frac{D}{1-D}\left(j_{0}\right)^{1-(1 / D)}=\sum_{j>j_{0}} l_{j}> & \sum_{j=j_{0}+1}^{j_{1}}\left(L+\frac{1}{2} \theta\right) j^{-1 / D}+\sum_{j=j_{1}+1}^{j_{2}-1} L j^{-1 / D}+\sum_{j=j_{2}}^{\infty} l_{j} \\
> & L \int_{j_{0}+1}^{j_{2}} t^{-1 / D} d t+\frac{1}{2} \theta \int_{j_{0}+1}^{j_{1}} t^{-1 / D} d t+\sum_{j=j_{2}+1}^{\infty} l_{j} \\
= & L \frac{D}{1-D}\left(\left(j_{0}+1\right)^{1-(1 / D)}-\left(j_{2}\right)^{1-(1 / D)}\right) \\
& +\frac{1}{2} \theta \frac{D}{1-D}\left(\left(j_{0}+1\right)^{1-(1 / D)}-\left(j_{1}\right)^{1-(1 / D)}\right) \\
& +\beta_{j_{2}} \frac{D}{1-D}\left(j_{2}\right)^{1-(1 / D)} .
\end{aligned}
$$

Therefore,

$$
\begin{aligned}
\beta_{j_{0}}> & L\left(\left(\frac{j_{0}}{j_{0}+1}\right)^{(1 / D)-1}-\left(\frac{j_{0}}{j_{2}}\right)^{(1 / D)-1}\right) \\
& +\frac{1}{2} \theta\left(\left(\frac{j_{0}}{j_{0}+1}\right)^{(1 / D)-1}-\left(\frac{j_{0}}{j_{1}}\right)^{(1 / D)-1}\right)+\beta_{j_{2}}\left(\frac{j_{0}}{j_{2}}\right)^{(1 / D)-1} .
\end{aligned}
$$


Since $\beta_{j_{2}} \rightarrow L$, we thus have

$$
\beta_{j_{0}} \geqslant L+\frac{1}{2} \theta\left(1-\left(r_{\theta}\right)^{(1 / D)-1}\right)+o(1) .
$$

This contradicts (4.4) if $j_{1}$ is sufficiently large (which, of course, forces $j_{0}$ to be large). Thus we deduce that

$$
\limsup _{j \rightarrow \infty} \alpha_{j} \leqslant L .
$$

To conclude the proof of Step 1 , we need to show that $\liminf _{j \rightarrow \infty} \alpha_{j} \geqslant L$. Suppose not. Then there are some $\theta$ with $0<\theta<1$ and an infinite set $S$ of subscripts $j$ with $\alpha_{j} \leqslant L(1-\theta)$ for all $j \in S$. Let

$$
\varepsilon=\theta-\frac{1-\theta}{D}\left((1-\theta)^{-D}-1\right) \text {. }
$$

Since $(1-\theta)^{1-D}<1-(1-D) \theta$ and $(1-\theta)^{-D}>1$, it easily follows that $0<$ $\varepsilon<\theta$.

For each large $j_{1} \in S$, there is a unique $j_{0}=j_{0}\left(j_{1}\right)$ such that

$$
j_{0}<j_{1}, \quad \alpha_{j_{0}}>L(1-\varepsilon), \quad \text { and } \quad \text { if } j_{0}<j<j_{1}, \text { then } \alpha_{j} \leqslant L(1-\varepsilon) .
$$

Indeed, the existence of $j_{0}$ for all large $j_{1} \in S$ follows from the second inequality in (4.5). For any $j_{2}>j_{1}$, we have

$$
\begin{aligned}
\beta_{j_{0}} \frac{D}{1-D}\left(j_{0}\right)^{1-(1 / D)}= & \sum_{j>j_{0}} l_{j}=\sum_{j=j_{0}+1}^{j_{1}} l_{j}+\sum_{j=j_{1}+1}^{j_{2}} l_{j}+\sum_{j>j_{2}} l_{j} \\
\leqslant & L(1-\varepsilon) \sum_{j=j_{0}+1}^{j_{1}} j^{-1 / D}+\left(j_{2}-j_{1}\right) l_{j_{1}}+\beta_{j_{2}} \frac{D}{1-D}\left(j_{2}\right)^{1-(1 / D)} \\
\leqslant & L(1-\varepsilon) \frac{D}{1-D}\left(\left(j_{0}\right)^{1-(1 / D)}-\left(j_{1}\right)^{1-(1 / D)}\right) \\
& +L(1-\theta)\left(j_{2}-j_{1}\right)\left(j_{1}\right)^{-(1 / D)}+\beta_{j_{2}} \frac{D}{1-D}\left(j_{2}\right)^{1-(1 / D)}
\end{aligned}
$$

Therefore,

$$
\begin{aligned}
\beta_{j_{0}} \leqslant L(1-\varepsilon)\left(1-\left(\frac{j_{0}}{j_{1}}\right)^{(1 / D)-1}\right) & +L(1-\theta)\left(\frac{j_{2}}{j_{1}}-1\right)\left(\frac{j_{0}}{j_{1}}\right)^{(1 / D)-1} \frac{1-D}{D} \\
& +\beta_{j_{2}}\left(\frac{j_{1}}{j_{2}}\right)^{(1 / D)-1}\left(\frac{j_{0}}{j_{1}}\right)^{(1 / D)-1} .
\end{aligned}
$$

We now choose $j_{2}=\left[(1-\theta)^{-D} j_{1}\right]$. Using (4.4), we have

$$
\begin{aligned}
\beta_{j_{0}} \leqslant & L(1-\varepsilon)+(L+o(1))\left(\frac{j_{0}}{j_{1}}\right)^{(1 / D)-1} \\
& \times\left(-(1-\varepsilon)+(1-\theta)\left((1-\theta)^{-D}-1\right) \frac{1-D}{D}+(1-\theta)^{1-D}\right),
\end{aligned}
$$

where the ' $o(1)$ ' tends to 0 as $j_{1} \rightarrow \infty$ through $S$.

The large parenthetical expression in (4.7) is

$$
-1+\varepsilon+(1-\theta)\left(\frac{(1-\theta)^{-D}-1}{D}+1\right)=-\theta+\varepsilon+\frac{1-\theta}{D}\left((1-\theta)^{-D}-1\right)=0,
$$


by our choice of $\varepsilon$. Thus from (4.7), we deduce that

$$
\beta_{j_{0}} \leqslant L(1-\varepsilon)+o(1) .
$$

Now, from the definition of $j_{0}$ and the assumption $l_{j+1} / l_{j} \rightarrow 1$, we have $\alpha_{j_{0}}=L(1-\varepsilon)+o(1)$. Consequently,

$$
\left(\alpha_{j_{0}}\right)^{D}+\left(\alpha_{j_{0}}\right)^{D-1} \beta_{j_{0}} \frac{D}{1-D} \leqslant \frac{L^{D}(1-\varepsilon)^{D}}{1-D}+o(1),
$$

contradicting (4.3) as $j_{1} \rightarrow \infty, j_{1} \in S$. Hence $S$ is not infinite and we conclude that $\lim \inf _{j \rightarrow \infty} \alpha_{j} \geqslant L$. Together with (4.6), this shows, as desired, that $\alpha_{j} \rightarrow L$, as $j \rightarrow \infty$ (that is, $l_{j} \sim L j^{-1 / D}$ ) and concludes the proof of Step 1.

Step 2. We now show that we may reduce the general case to the situation where $\left(l_{j}\right)_{j=1}^{\infty}$ is strictly decreasing and $l_{j+1} / l_{j} \rightarrow 1$. To this end, let $\left(l_{j}\right)_{j=1}^{\infty}$ be an arbitrary sequence satisfying the hypothesis of the theorem.

If $l_{j}>l_{j+1}$, we have $J\left(l_{j}\right)=j$ and

$$
2\left(\frac{1}{2} l_{j}\right)^{D} J\left(l_{j}\right)=2^{1-D}\left(\alpha_{j}\right)^{D} .
$$

We conclude from (3.2) and (4.1) that

$$
\limsup _{j: l_{j}>l_{j+1}} \alpha_{j} \leqslant L(1-D)^{-1 / D} \text {. }
$$

If $l_{j}=l_{j+1}=\ldots=l_{j_{0}}>l_{j_{0}+1}$, then $\alpha_{j}<\alpha_{j+1}<\ldots<\alpha_{j_{0}}$, so that

$$
\limsup _{j \rightarrow \infty} \alpha_{j} \leqslant L(1-D)^{-1 / D} \text {. }
$$

We now show that the hypothesis of the theorem forces

$$
\lim _{j \rightarrow \infty} \frac{l_{j}}{l_{j+1}}=1 \text {, }
$$

which is one of the two facts we need to complete the proof.

Suppose $l_{j} \geqslant \varepsilon>l_{j+1}$. Then $J(\varepsilon)=j$. Writing $\varepsilon=r l_{j}$, where $1 \geqslant r>l_{j+1} / l_{j}$, we have

$$
\begin{aligned}
L_{D}\left(\frac{1}{2} \varepsilon\right)=2\left(\frac{1}{2} \varepsilon\right)^{D} j+\left(\frac{1}{2} \varepsilon\right)^{D-1} \sum_{k>j} l_{j} & =2^{1-D} r^{D}\left(l_{j}\right)^{D} j+2^{1-D} r^{D-1}\left(l_{j}\right)^{D-1} \sum_{k>j} l_{j} \\
& =r^{D} A_{j}+r^{D-1} B_{j}, \quad \text { say. }
\end{aligned}
$$

Then $A_{j}+B_{j}=L_{D}\left(\frac{1}{2} l_{j}\right) \rightarrow 2^{1-D} L^{D} /(1-D)$, as $j \rightarrow \infty$ through numbers with $l_{j}>$ $l_{j+1}$. Since each $A_{j}, B_{j}>0$, the set $\left\{\left(A_{j}, B_{j}\right): l_{j}>l_{j+1}\right\}$ is contained in some compact subset $K$ of $\mathbb{R}^{2}-\{(0,0)\}$.

Suppose there is some $\theta \in(0,1)$ such that $l_{j+1} / l_{j}<1-\theta$ for infinitely many $j$. For any fixed $A, B$, let $\omega(A, B)$ denote the difference between the maximum and minimum values of the function $r^{D} A+r^{D-1} B$ on the compact interval $[1-\theta, 1]$. It is an elementary exercise that $\omega(A, B)$ is continuous and positive on $\mathbb{R}^{2}-\{(0,0)\}$. Hence it assumes a positive ininimum $\eta$ on the compact set $K$. Thus for each $j$ with $l_{j+1} / l_{j}<1-\theta$, there are $r_{1}, r_{2} \in[1-\theta, 1]$ with

$$
L_{D}\left(\frac{1}{2} r_{1} l_{j}\right)-L_{D}\left(\frac{1}{2} r_{2} l_{j}\right) \geqslant \eta>0 .
$$

This contradicts (4.1), so we have (4.9). 
For any real number $\varepsilon>0$ and $j \in \mathbb{N}$, define $L_{D}(\varepsilon, j)$ by

$$
L_{D}(\varepsilon, j):=2 \varepsilon^{D} j+\varepsilon^{D-1} \sum_{k>j} l_{k} .
$$

For example, $L_{D}(\varepsilon, J(2 \varepsilon))=L_{D}(\varepsilon)$, with $L_{D}(\varepsilon)$ given by (3.2). We now show that if $j_{\varepsilon}$ is any integer with $l_{j_{\varepsilon}}=l_{J_{(2 \varepsilon)}}$, then no matter how $j_{\varepsilon}$ is chosen for each $\varepsilon>0$, we have

$$
\lim _{\varepsilon \rightarrow 0^{+}} L_{D}\left(\varepsilon, j_{\varepsilon}\right)=\lim _{\varepsilon \rightarrow 0^{+}} L_{D}(\varepsilon) \text {. }
$$

Given $\varepsilon>0$, write $j=j_{\varepsilon}$ and $j_{0}=J(2 \varepsilon)$. Then

Thus

$$
\begin{aligned}
L_{D}(\varepsilon, j)-L_{D}(\varepsilon) & =2 \varepsilon^{D}\left(j-j_{0}\right)+\varepsilon^{D-1} \sum_{k=j+1}^{j_{0}} l_{k} \\
& =\left(j_{0}-j\right)\left(-2 \varepsilon^{D}+\varepsilon^{D-1} l_{j_{0}}\right) \\
& =\varepsilon^{D-1}\left(j_{0}-j\right)\left(-2 \varepsilon+l_{j_{0}}\right) .
\end{aligned}
$$

$$
0 \leqslant L_{D}(\varepsilon, j)-L_{D}(\varepsilon) \leqslant \varepsilon^{D-1} j_{0}\left(-2 \varepsilon+l_{j_{0}}\right) .
$$

This last expression is a decreasing function of $\varepsilon$ for $\varepsilon>\left(\frac{1}{2}-\frac{1}{2} D^{-1}\right) l_{j_{0}}$. From (4.9), if $\varepsilon$ is sufficiently small (so that $j_{0}$ is large), we have

$$
\varepsilon>\frac{1}{2} l_{j_{0}+1}>\left(\frac{1}{2}-\frac{1}{2} D^{-1}\right) l_{j_{0}} .
$$

Thus replacing $\varepsilon$ with $\frac{1}{2} l_{j_{0}+1}$ makes the expression larger, so that

$$
\begin{aligned}
0 \leqslant L_{D}(\varepsilon, j)-L_{D}(\varepsilon) & <2^{1-D}\left(l_{j_{0}+1}\right)^{D-1} j_{0}\left(-l_{j_{0}+1}+l_{j_{0}}\right) \\
& =2^{1-D}\left(l_{j_{0}+1}\right)^{D} j_{0}\left(-1+\frac{l_{j_{0}}}{l_{j_{0}+1}}\right) \\
& =2^{1-D}\left(\alpha_{j_{0}+1}\right)^{D} \frac{j_{0}}{j_{0}+1}\left(-1+\frac{l_{j_{0}}}{l_{j_{0}+1}}\right) .
\end{aligned}
$$

Thus (4.8) and (4.9) imply (4.10).

We now define a sequence $\left(m_{j}\right)_{j=1}^{\infty}$ of non-negative reals with the following properties. We let $m_{1}=0$. If $l_{j}>l_{j+1}$, we let $m_{j+1}=0$. If

$$
l_{j}=l_{j+1}=\ldots=l_{j+k}>l_{j+k+1},
$$

we let $0<m_{j+1}<m_{j+2}<\ldots<m_{j+k}<l_{j+k+1}-l_{j+k}$. Further, we choose the positive $m$ values so small that, as $j \rightarrow \infty$,

$$
\sum_{q>j} m_{q}=o\left(\left(l_{j}\right)^{1-D}\right) \text { and } m_{j}=o\left(l_{j}\right)
$$

Then the positive sequence $\left(l_{j}-m_{j}\right)_{j=1}^{\infty}$ is strictly decreasing and $l_{j} \sim L j^{-1 / D}$ if and only if $l_{j}-m_{j} \sim L j^{-1 / D}$, as $j \rightarrow \infty$.

We now show that $\left(l_{j}-m_{j}\right)_{j=1}^{\infty}$ satisfies the same hypothesis $(4.1)$ as $\left(l_{j}\right)_{j=1}^{\infty}$, thus completing our proof. Let $J^{\prime}(\varepsilon)$ be the analogue of the function $J(\varepsilon)$ for the sequence $\left(l_{j}-m_{j}\right)_{j=1}^{\infty}$. We need to prove that

$$
L_{D}^{\prime}(\varepsilon):=2 \varepsilon^{D} J^{\prime}(2 \varepsilon)+\varepsilon^{D-1} \sum_{j>J^{\prime}(2 \varepsilon)}\left(l_{j}-m_{j}\right)
$$

satisfies $L_{D}^{\prime}(\varepsilon) \rightarrow 2^{1-D} L^{D} /(1-D)$, as $\varepsilon \rightarrow 0^{+}$. To this end, it will suffice to show that $L_{D}^{\prime}(\varepsilon)-L_{D}(\varepsilon) \rightarrow 0$. 
From the above construction, we have $l_{J(\varepsilon)}=l_{J^{\prime}(\varepsilon)}$ for any $\varepsilon>0$. Indeed, since $l_{j}-m_{j} \leqslant l_{j}$, we have $J^{\prime}(\varepsilon) \leqslant J(\varepsilon)$. If $J^{\prime}(\varepsilon)<J(\varepsilon)$, then since

$$
l_{J^{\prime}(\varepsilon)}-m_{J^{\prime}(\varepsilon)} \geqslant \varepsilon>l_{j}-m_{j}
$$

for all $j>J^{\prime}(\varepsilon)$ and since $l_{J(\varepsilon)} \geqslant \varepsilon$, we have $m_{j}>0$ for $j=J^{\prime}(\varepsilon)+1, \ldots, J(\varepsilon)$. Thus $l_{J^{\prime}(\varepsilon)}=l_{J(\varepsilon)}$.

We write

$$
L_{D}^{\prime}(\varepsilon)-L_{D}(\varepsilon)=\left(L_{D}^{\prime}(\varepsilon)-L_{D}\left(\varepsilon, J^{\prime}(2 \varepsilon)\right)\right)+\left(L_{D}\left(\varepsilon, J^{\prime}(2 \varepsilon)\right)-L_{D}(\varepsilon)\right) .
$$

Now, by (4.10), $L_{D}\left(\varepsilon, J^{\prime}(2 \varepsilon)\right)-L_{D}(\varepsilon) \rightarrow 0$ as $\varepsilon \rightarrow 0^{+}$. Further,

$$
\begin{aligned}
\left|L_{D}^{\prime}(\varepsilon)-L_{D}\left(\varepsilon, J^{\prime}(2 \varepsilon)\right)\right| & =\varepsilon^{D-1} \sum_{q>J^{\prime}(2 \varepsilon)} m_{q} \\
& =o\left(\varepsilon^{D^{-1}}\left(l_{J^{\prime}(2 \varepsilon)}\right)^{1-D}\right)=o\left(\varepsilon^{D-1}\left(l_{J(2 \varepsilon)}\right)^{1-D}\right),
\end{aligned}
$$

by (4.11). Further, by (4.9), $l_{J(2 \varepsilon)} \sim 2 \varepsilon$ as $\varepsilon \rightarrow 0^{+}$. Thus the above expression is $o(1)$. We conclude from (4.12) that $L_{D}^{\prime}(\varepsilon)-L_{D}(\varepsilon) \rightarrow 0$, as $\varepsilon \rightarrow 0^{+}$, which completes our proof of Theorem 4.1.

\subsection{Existence of a second term: the Riemann zeta-function}

We conclude here the proof of Corollary 2.3 (Conjecture 1 in the case where $n=1$ ) by proving Theorem 2.1 , which we restate as follows:

Theorem 4.2. Suppose $l_{1} \geqslant l_{2} \geqslant \ldots>0$ and

$$
l_{j} \sim L j^{-1 / D}, \text { as } j \rightarrow \infty, \text { for } L>0 \text { and } D \in(0,1) \text {. }
$$

Let $\delta(x)=\sum_{j=1}^{\infty}\left\{l_{j} x\right\}$, as in (3.13). Then

$$
\delta(x) \sim-\zeta(D) L^{D} x^{D}, \quad \text { as } x \rightarrow+\infty,
$$

where $\zeta=\zeta(s)$ denotes the Riemann zeta-function.

Proof. Let $J(\varepsilon)$ be defined by (3.1). We easily deduce from (4.13) that

$$
J(\varepsilon) \sim L^{D} \varepsilon^{-D}, \quad \text { as } \varepsilon \rightarrow 0^{+} .
$$

Let $k \geqslant 2$ be an arbitrary fixed integer. Using $J(1 / x) \geqslant J(2 / x) \geqslant \ldots$, the fact that $J(q / x)<j \leqslant J((q-1) / x)$ implies $\left[l_{j} x\right]=q-1$, and various elementary rearrangements of sums, including partial summation, we obtain

$$
\begin{aligned}
\delta(x) & =x \sum_{j>J(1 / x)} l_{j}+\sum_{j \leqslant J(k / x)}\left\{l_{j} x\right\}+\sum_{q=2}^{k} \sum_{j=J(q / x)+1}^{J((q-1) / x)}\left\{l_{j} x\right\} \\
& =x \sum_{j>J(1 / x)} l_{j}+\sum_{j \leqslant J(k / x)}\left\{l_{j} x\right\}+\sum_{q=2}^{k} \sum_{j=J(q / x)+1}^{J((q-1) / x)}\left(l_{j} x-(q-1)\right) \\
& =x \sum_{j>J(k / x)} l_{j}+\sum_{j \leqslant J(k / x)}\left\{l_{j} x\right\}-\sum_{q=2}^{k}(q-1)\left(J\left(\frac{q-1}{x}\right)-J\left(\frac{q}{x}\right)\right) \\
& =x \sum_{j>J(k / x)} l_{j}+\sum_{j \leqslant J(k / x)}\left\{l_{j} x\right\}-\sum_{q=1}^{k-1} J\left(\frac{q}{x}\right)+(k-1) J\left(\frac{k}{x}\right) .
\end{aligned}
$$

In the light of (4.16), we can write

$$
\delta(x)=A+B+C,
$$


where

$$
\begin{aligned}
& A:=x \sum_{j>J(k / x)} l_{j} . \\
& B:=k J\left(\frac{k}{x}\right)-\sum_{q=1}^{k-1} J\left(\frac{q}{x}\right)
\end{aligned}
$$

and

$$
C:=\sum_{j \leqslant J(k / x)}\left(\left\{l_{j} x\right\}-1\right) .
$$

Note that in (4.17) and (4.18), $A, B$ and $C$ depend on $x$. We now estimate, for fixed $k$, each of these terms, as $x \rightarrow+\infty$.

We first observe that since $-1 \leqslant\left\{l_{j} x\right\}-1<0$, we have $-J(k / x) \leqslant C \leqslant 0$; it thus follows from (4.15) that (for fixed $k$ ),

$$
0 \leqslant-(L x)^{-D} C \leqslant(L x)^{-D} J(k / x) \rightarrow k^{-D}, \quad \text { as } x \rightarrow+\infty .
$$

Next, we deduce from (4.15) and (4.18b) that

$$
(L x)^{-D} B \rightarrow k^{1-D}-\sum_{q=1}^{k-1} q^{-D}, \quad \text { as } x \rightarrow+\infty .
$$

Further, we claim that

$$
(L x)^{-D} A \rightarrow k^{1-D} \frac{D}{1-D}, \quad \text { as } x \rightarrow+\infty ;
$$

indeed, according to (4.13) and (4.15), for each small $\varepsilon>0$, there is some $x_{0}>0$ such that for all $x \geqslant x_{0}$, we have $l_{j} \in\left((L-\varepsilon) j^{-1 / D},(L+\varepsilon) j^{-1 / D}\right)$ for all $j>J(k / x)$. Thus, by (4.18a), we have for all $x \geqslant x_{0}$,

$$
\begin{aligned}
A<x \sum_{j>J(k / x)}(L+\varepsilon) j^{-1 / D} & <x(L+\varepsilon) \int_{J(k / x)}^{+\infty} t^{-1 / D} d t \\
& =x(L+\varepsilon) \frac{D}{1-D}(J(k / x))^{1-(1 / D)} .
\end{aligned}
$$

Hence, by (4.15),

$$
A \leqslant(1+o(1)) k^{1-D} \frac{D}{1-D}(L+\varepsilon) L^{D-1} x^{D}, \quad \text { as } x \rightarrow+\infty .
$$

A similar computation shows that

$$
A \geqslant(1+o(1)) k^{1-D} \frac{D}{1-D}(L-\varepsilon) L^{D-1} x^{D}, \quad \text { as } x \rightarrow+\infty .
$$

Since $\varepsilon>0$ can be chosen arbitrarily small, we have (4.21).

Now, we combine (4.20) and (4.21) to obtain that for fixed $k$,

$$
(L x)^{-D}(A+B) \rightarrow \frac{1}{1-D} k^{1-D}-\sum_{q=1}^{k-1} q^{-D}=f_{k}(D)+\frac{1}{1-D}, \quad \text { as } x \rightarrow+\infty,
$$

where

$$
f_{k}(s):=\int_{1}^{k}\left(t^{-s}-[t]^{-s}\right) d t \quad\left(=-\frac{1}{1-s}+\frac{1}{1-s} k^{1-s}-\sum_{q=1}^{k-1} q^{-s}\right) .
$$


The sequence of entire functions $\left\{f_{k}(s)\right\}_{k=1}^{\infty}$ converges uniformly as $k \rightarrow \infty$ on every compact subset of $\operatorname{Re} s>0$ to the function

$$
f(s):=\int_{1}^{+\infty}\left(t^{-s}-[t]^{-s}\right) d t,
$$

which is thus analytic in the domain $\operatorname{Re} s>0$. Further, as noted in (2.3), we have

$$
f(s)=-\frac{1}{1-s}-\zeta(s), \quad \text { for } \operatorname{Re} s>0
$$

Hence

$$
f_{k}(D)+\frac{1}{1-D} \rightarrow-\zeta(D), \quad \text { as } k \rightarrow \infty .
$$

From (4.17), (4.19) and (4.22), we deduce that for each fixed $k \geqslant 2$,

$$
\begin{aligned}
-k^{-D}+f_{k}(D)+\frac{1}{1-D} & \leqslant \liminf _{x \rightarrow+\infty}(L x)^{-D} \delta(x) \\
& \leqslant \limsup _{x \rightarrow+\infty}(L x)^{-D} \delta(x) \leqslant f_{k}(D)+\frac{1}{1-D} .
\end{aligned}
$$

By letting $k \rightarrow \infty$ and using (4.24), we obtain $(L x)^{-D} \delta(x) \rightarrow-\zeta(D)$ as $x \rightarrow+\infty$; that is, (4.14) holds, as desired.

\subsection{Examples}

We illustrate our main results with two instructive examples. The first is actually a family of examples that illustrate Conjecture 1 (equivalent to Corollary 2.3) for all values of $D \in(0,1)$. Working out the details for this family of examples (with expert help on the computer from Mr Vivek Shivpuri) actually led us to our main results in this paper. This family was also used to illustrate the error estimates (in the case where $n=1$ ) in [16]. The second example is the complement in $[0,1]$ of everybody's 'favourite fractal' in dimension one, namely the ternary Cantor set. This example illustrates Conjecture 1 ' and its converse (see Theorem 2.4) and, in particular, shows that these results are the best possible.

Example 4.3 (cf. [16, Example 5.1, pp. 512-514]). Let $a>0$ be fixed, but arbitrary. Let $\Omega=\Omega(a)=\bigcup_{j=1}^{\infty} I_{j}$, where $I_{j}=\left((j+1)^{-a}, j^{-a}\right)$. Thus $\Omega$ is a dense open subset of $[0,1]$. We have $\Gamma=\partial \Omega=\left\{j^{-a}: j \geqslant 1\right\} \cup\{0\}$ and

$$
l_{j}=j^{-a}-(j+1)^{-a} \sim a j^{-a-1},
$$

as $j \rightarrow \infty$. Thus (2.4) is satisfied with $L=a$ and $D=(a+1)^{-1}$. It follows from Theorem 2.2 (or, for the present example, from [16, Theorem C of Appendix C, pp. 523-524]) that $\Gamma$ is Minkowski measurable and

$$
\mathcal{M}(D ; \Gamma)=\frac{2^{1-D}}{1-D} L^{D}=(a+1)\left(\frac{2}{a}\right)^{a /(a+1)} .
$$

In addition, we deduce from Corollary 2.3 that (not only does $\varphi(\lambda)-N(\lambda) \approx \lambda^{D / 2}$, as $\lambda \rightarrow+\infty$, as was shown for this example in [16, equation (5.8), p. 513], but also 
that) the asymptotic expansion of $N(\lambda)$ admits a second term, given by

$$
\begin{aligned}
N(\lambda) & =\pi^{-1} \lambda^{\frac{1}{2}}-c_{1, D} M(D ; \Gamma) \lambda^{D / 2}+o\left(\lambda^{D / 2}\right) \\
& =\pi^{-1} \lambda^{\frac{1}{2}}+\zeta(D)(a / \pi)^{D} \lambda^{D / 2}+o\left(\lambda^{D / 2}\right),
\end{aligned}
$$

as $\lambda \rightarrow+\infty$. (Since $|\Omega|_{1}=1$ in this case, we have $\varphi(\lambda)=\pi^{-1} \lambda^{\frac{1}{2}}$ here.)

Remark 4.4. Recall from [16] that since $\Gamma$ is countable, we have $H=0$ for every $a>0$ and thus Berry's original conjecture [1,2] (expressed in terms of the Hausdorff dimension $H$ rather than the Minkowski dimension $D$ of $\Gamma$ ) clearly fails for this family of examples. As was pointed out in [16, Remarks 5.2(a), (b), p. 514], this is due to the fact that the Hausdorff measure is countably additive whereas the pre-measure induced by the Minkowski content is only finitely sub-additive.

Example 4.5 (the Cantor set). Consider now the set $\Gamma$ of $x \in[0,1]$ which have a ternary expansion devoid of ones. This is the familiar ternary Cantor set. It is uncountable, compact and has (one-dimensional) Lebesgue measure 0 . If $\Omega$ is the complement of $\Gamma$ in $[0,1]$, we have that $\Omega \subset \mathbb{R}$ is open and dense in $[0,1]$ with $\partial \Omega=\Gamma$. The sequence $\left(l_{j}\right)_{j=1}^{\infty}$ of interval lengths of the components of $\Omega$ is

$$
\frac{1}{3}, \frac{1}{9}, \frac{1}{9}, \frac{1}{27}, \frac{1}{27}, \frac{1}{27}, \frac{1}{27}, \ldots \text {, }
$$

where we have $2^{k-1}$ copies of $3^{-k}$ for $k=1,2, \ldots$. We now compute $D, \alpha, \beta, \delta_{*}, \delta^{*}, \mu_{*}, \mu^{*}$ for $\Gamma=\partial \Omega$ (that is, for the sequence $\left.\left(l_{j}\right)_{j=1}^{\infty}\right)$. In particular, we shall see that $0<\alpha<\beta<+\infty$ and the same relationship holds for $\delta_{*}, \delta^{*}$ and for $\mathcal{M}_{*}, \mathcal{M}^{*}$. Thus, in some sense, Theorem 2.4 is the best possible.

THEOREM 4.6. The sequence $\left(l_{j}\right)_{j=1}^{\infty}$, where we have $2^{k-1}$ copies of $3^{-k}$ for $k=1,2, \ldots$, satisfies $D=\ln 2 / \ln 3, \alpha=\frac{1}{3}, \beta=1, \delta_{*}=1, \delta^{*}=2$,

$$
\mu_{*}=2^{1-D}\left(\left(\frac{1-D}{D}\right)^{D}+\left(\frac{1-D}{D}\right)^{-(1-D)}\right)=\frac{\ln 9}{\ln \frac{3}{2}}\left(\frac{\ln \frac{3}{2}}{\ln 4}\right)^{\ln 2 / \ln 3}
$$

and

$$
M^{*}=2^{2-D}=2^{2-(\ln 2 / \ln 3)} .
$$

Proof. Let

$$
j=j(k)=1+2+\ldots+2^{k-1}=2^{k}-1 .
$$

Thus $j \sim 2^{k}$ as $k \rightarrow \infty$. We have $l_{j}=3^{-k}, l_{j+1}=3^{-k-1}$. Hence

$$
l_{j} j^{j \ln 3 / \ln 2} \sim 1 \text { and } l_{j+1}(j+1)^{\ln 3 / \ln 2} \sim \frac{1}{3},
$$

as $k \rightarrow \infty$. It is clear now that $D=\ln 2 / \ln 3, \alpha=\frac{1}{3}$ and $\beta=1$.

We now compute $\delta_{*}$ and $\delta^{*}$. Let $x$ be large and let $k$ be a positive integer with $3^{k} \leqslant x<3^{k+1}$. Then

$$
\begin{aligned}
x^{-D} \delta(x) & =x^{-D} \sum_{l_{j} x \geqslant 1}\left\{l_{j} x\right\}+x^{1-D} \sum_{l_{j} x<1} l_{j} \\
& =x^{-D} \sum_{i=1}^{k} 2^{i-1}\left\{3^{-i} x\right\}+x^{1-D} \sum_{i=k+1}^{\infty} 2^{i-1} 3^{-i} \\
& =x^{-D} \sum_{i=1}^{k} 2^{i-1}\left\{3^{-i} x\right\}+x^{1-D}\left(\frac{2}{3}\right)^{k} .
\end{aligned}
$$


The first term at the end of equation (4.26) is non-negative and the second is minimized for $x$ in $\left[3^{k}, 3^{k+1}\right)$ at $x=3^{k}$. But the first term is 0 at $x=3^{k}$, so for $x \in\left[3^{k}, 3^{k+1}\right), x^{-D} \delta(x)$ is minimized when $x=3^{k}$ and the value there is exactly 1 (using $D=\ln 2 / \ln 3$ and hence $3^{D}=2$ ). Thus $\delta_{*}=1$.

The expression at the end of equation (4.26) is less than

$$
x^{-D} \sum_{i=1}^{k} 2^{i-1}+x^{1-D}\left(\frac{2}{3}\right)^{k}=x^{-D}\left(2^{k}-1\right)+x^{1-D}\left(\frac{2}{3}\right)^{k}
$$

that is, we have just replaced each $\left\{3^{-i} x\right\}$ with 1 . Thus

$$
x^{-D} \delta(x)<x^{-D} 2^{k}+x^{1-D}\left(\frac{2}{3}\right)^{k},
$$

for all $x$ in $\left[3^{k}, 3^{k+1}\right)$. For $x$ in $\left[3^{k}, 3^{k+1}\right]$, the expression on the right-hand side of (4.27) is maximized at the end points of this interval and has the value 2 at both end points (again using $D=\ln 2 / \ln 3$ ). Thus $\delta^{*} \leqslant 2$. Further, by choosing $x=3^{k+1}-3^{-k}$, we have each $\left\{3^{-i} x\right\}$ in equation (4.26) nearly equal to 1 ; it is $1-3^{-k-i}$. For this value of $x$, we have $x^{D}=2^{k+1}\left(1+O\left(3^{-2 k}\right)\right)$. Thus we deduce from equation (4.26) that

$$
\begin{aligned}
x^{-D} \delta(x) & =2^{-k-1}\left(1+O\left(3^{-2 k}\right)\right)\left(2^{k}+O(1)\right)+\left(\frac{3}{2}\right)^{k+1}\left(1+O\left(3^{-2 k}\right)\right)\left(\frac{2}{3}\right)^{k} \\
& =2+o(1), \quad \text { as } k \rightarrow \infty .
\end{aligned}
$$

We conclude that $\delta^{*}=2$.

Finally, we compute $\mathcal{M}_{*}$ and $\mu^{*}$. Suppose $\varepsilon>0$ is small. Let $k$ be a positive integer with $3^{-k} \geqslant 2 \varepsilon>3^{-k-1}$. Then

$$
J(2 \varepsilon)=1+2+\ldots+2^{k-1}=2^{k}-1 .
$$

Further,

$$
\sum_{j>J(2 \varepsilon)} l_{j}=\sum_{i=k+1}^{\infty} 2^{i-1} 3^{-i}=\left(\frac{2}{3}\right)^{k} .
$$

Thus, recalling (3.2), we have

$$
\begin{aligned}
L_{D}(\varepsilon)=2 \varepsilon^{D} J(2 \varepsilon)+\varepsilon^{D-1} \sum_{j>J(2 \varepsilon)} l_{j} & =2 \varepsilon^{D}\left(2^{k}-1\right)+\varepsilon^{D-1}\left(\frac{2}{3}\right)^{k} \\
& =\varepsilon^{D} 2^{k+1}+\varepsilon^{D-1}\left(\frac{2}{3}\right)^{k}+o(1),
\end{aligned}
$$

as $\varepsilon \rightarrow 0^{+}$. The expression $\varepsilon^{D} 2^{k+1}+\varepsilon^{D-1}\left(\frac{2}{3}\right)^{k}$ is minimized for $2 \varepsilon$ in $\left(3^{-k-1}, 3^{-k}\right]$ at $\varepsilon=(1-D) /\left(2 D .3^{k}\right)$ and has the value (using $D=\ln 2 / \ln 3$ )

$$
2^{1-D}\left(\left(\frac{1-D}{D}\right)^{D}+\left(\frac{1-D}{D}\right)^{-(1-D)}\right)
$$

there. Further, it is maximized at $3^{-k} / 2$ (that is, at the right-hand end point) and has the value $2^{2-D}$ there. This concludes our computation of $\mathcal{M}_{*}$ and $\mathcal{M}^{*}$.

Remarks 4.7. (a) According to Theorem 4.6, $0<\mu_{*}<\mu^{*}<+\infty$ and thus the Cantor set $\Gamma=\partial \Omega$ is not Minkowski measurable; further, $0<\delta_{*}<\delta^{*}<+\infty$ and hence the asymptotic expansion of $N(\lambda)$ does not admit a monotonic second term (that is, $\lambda^{-D / 2}(N(\lambda)-\varphi(\lambda))$ does not converge). Consequently, the error estimates of [16] (or of Theorem 3.10) are optimal and cannot be improved in this case; further, this example shows that the hypothesis of Minkowski measurability is necessary in the statement of Conjecture 1. 
(b) Intuitively-and paradoxically in view of the comments made in [1] - the fact that $N(\lambda)$ does not admit an asymptotic second term is due to the symmetry of the boundary $\Gamma$ (here, the self-similarity of the Cantor set). Indeed, this symmetry gives rise to high multiplicities in the eigenvalues (equivalently, in the interval lengths $\left.\left(l_{j}\right)_{j=1}^{\infty}\right)$ and thus causes the function $\lambda^{-D / 2}(N(\lambda)-\varphi(\lambda))$ to oscillate.

(c) Since the Cantor set $\Gamma$ is self-similar and satisfies the 'open set condition', its Minkowski and Hausdorff dimensions coincide: $D=H=\ln 2 / \ln 3$, the 'similarity dimension' of $\Gamma$. (See, for example, [25, Theorem 4.19, p. 20].)

\subsection{Remarks}

We close this section with two related comments. As should be clear to the reader, the second one is of a purely speculative nature.

4.4.a. Let $\hat{M}=\hat{M}(D ; \Gamma)$ be the normalized Minkowski content of $\Gamma$. (If $A \subset \mathbb{B}^{n}, \hat{M}(d ; A):=\mathcal{M}(d ; A) / \mathscr{B}_{n-d}$, with $\mathscr{B}_{m}:=\pi^{m / 2} / \Gamma\left(1+\frac{1}{2} m\right)$, even if $m$ is not an integer; here, $\Gamma(s)$ denotes the classical gamma function. When $d$ is an integer, this normalization is standard in geometric measure theory (see [9, p. 273]); in this case, when $A$ is 'sufficiently regular' (that is, ' $d$-rectifiable' [9, pp. 251, 275]), then $\hat{M}(d ; A)=|A|_{d}$, the (ordinary) $d$-dimensional Lebesgue measure of $A$.) Then, in (2.9) (and in (4.25) above), we have

$$
c_{1, D} \mathcal{M}(D ; \Gamma)=\Lambda(D) \hat{M}(D ; \Gamma),
$$

where

$$
\Lambda(s):=\left(\frac{1}{2} \pi\right)^{-s} \pi^{(1-s) / 2} \frac{1}{\Gamma\left(\frac{1}{2}(1-s)\right)}(-\zeta(s)), \quad \text { for } s \in \mathbb{C} .
$$

The function $\Lambda=\Lambda(s)$ (discovered in this somewhat surprising manner) is entire and satisfies the functional equation (equivalent to that of $\zeta$ (see, for example, [32, $\S \mathrm{II}])$ )

$$
\left(\frac{1}{2} \pi\right)^{s} \Lambda(s)=\left(\frac{1}{2} \pi\right)^{1-s} \Lambda(1-s), \quad \text { for all } s \in \mathbb{C} .
$$

Moreover, $\Lambda$ has the same zeros as $\zeta$ in the 'critical strip' $0<\operatorname{Re} s<1$.

Finally, we note that clearly, even when $D=H$ and the Minkowski content is normalized as above, the associated normalized constant $\hat{c}_{1, D}:=\Lambda(D)$ does not coincide with the corresponding value of $\mathscr{C}_{1, H}$ (with $H=D$ ) conjectured by Berry in [1]. (Compare equations (1.9) and (4.29) above.)

4.4.b. Could there exist a notion of 'complex fractal dimension' which would enable us to obtain a natural interpretation of $\zeta$ and $\Lambda$ in the 'critical strip' $0<\operatorname{Re} s<1$ ? If so, we might extend to the complex domain the following observation: the vertical line $\operatorname{Re} s=0$ to the left of the 'critical strip' would correspond to the least 'fractal' (that is, 'non-fractal') case. Similarly, the vertical line $\operatorname{Re} s=1$ to the right of the 'critical strip' would correspond to the most 'fractal' case. Moreover, the symmetries of $\zeta$ and $\Lambda$ with respect to the 'critical line' $\operatorname{Re} s=\frac{1}{2}$ (the 'midfractal' case, in the sense of [16]) could naturally be reinterpreted in terms of the 'conjugate fractional exponents' $s$ and $1-s$ (introduced in [16, Remarks 4.10 and 5.3(a), pp. 507-508, 515] for $s \in[0,1]$ ). 


\section{Acknowledgements}

The first author is grateful to his programmer, Mr Vivek Shivpuri, the Advanced Computational Methods Center (ACMC) of the University of Georgia, as well as to the Department of Applied Mathematics of the Universite Blaise Pascal (Clermont II) in Clermont-Ferrand, France, where he was Professor Invite in June 1988 at the beginning of this work, and to the Department of Mathematics of Yale University where he was Visiting Professor during the completion of the manuscript.

The second author is grateful to the Institute for Advanced Study in Princeton, New Jersey, for its hospitality during the completion of work on this paper.

Part of this work was presented (by M.L.L.) at the special session on 'Geometric Spectral and Inverse Spectral Problems' of the Annual Meeting of the American Mathematical Society held in Louisville, Kentucky, in January 1990, as well as (see [19]) to the 'UAB International Conference on Mathematical Physics and Differential Equations' held in Birmingham, Alabama, in March 1990.

\section{References}

1. M. V. BERRY, 'Distribution of modes in fractal resonators', Structural stability in physics (eds W. Güttinger and H. Eikemeier, Springer, Berlin, 1979), pp. 51-53.

2. M. V. BERRY, 'Some geometric aspects of wave motion: wavefront dislocations, diffraction catastrophes, diffractals', Geometry of the Laplace operator (eds R. Osserman and A. Weinstein), Proceedings of Symposia in Pure Mathematics 36 (American Mathematical Society, Providence, R. I., 1980), pp. 13-38.

3. A. S. Besicovitch and S. J. TAYLOR, 'On the complementary intervals of a linear closed set of zero Lebesgue measure', J. London Math. Soc. 29 (1954) 449-459.

4. G. Bouligand, 'Ensembles impropres et nombre dimensionnel', Bull. Sci. Math. (2) 52 (1928) 320-344, 361-376.

5. H. BRÉzis, Analyse fonctionnelle: théorie et applications (Masson, Paris, 1983).

6. J. Brossard and R. Carmona, 'Can one hear the dimension of a fractal?', Comm. Math. Phys. 104 (1986) 103-122.

7. G. Cherbit (ed.), Fractals, dimensions non entières et applications (Masson, Paris, 1987).

8. Y. Dupain, M. Mendès-France, and C. Tricot, 'Dimension des spirales', Bull. Soc. Math. France 111 (1983) 193-201.

9. H. FEDERER, Geometric measure theory (Springer, Berlin, 1969).

10. J. HAWKES, 'Hausdorff measure, entropy, and the independence of small sets', Proc. London Math. Soc. (3) 28 (1974) 700-724.

11. L. HöRMANDER, 'The spectral function of an elliptic operator', Acta Math. 121 (1968) 193-218.

12. L. HöRMANDER, The analysis of linear partial differential operators, vols III, IV (Springer, Berlin, 1985).

13. V. JA. IVRII, 'Second term of the spectral asymptotic expansion of the Laplace-Beltrami operator on manifolds with boundary', Functional Anal. Appl. 14 (1980) 98-106.

14. V. JA. IVRII, Precise spectral asymptotics for elliptic operators acting in fiberings over manifolds with boundary, Lecture Notes in Mathematics 1100 (Springer, Berlin, 1984).

15. J.'-P. KAHANE and R. SAlem, Ensembles parfaits et séries trigonométriques (Hermann, Paris, 1963).

16. M. L. LapIDUS, 'Fractal drum, inverse spectral problems for elliptic operators and a partial resolution of the Weyl-Berry conjecture', Trans. Amer. Math. Soc. 325 (1991) 465-529.

17. M. L. LAPIDUs, 'Can one hear the shape of a fractal drum? Partial resolution of the Weyl-Berry conjecture', Geometric analysis and computer graphics, Proceedings, MSRI, Berkeley, May 1988 (eds P. Concus, R. Finn, and D. A. Hoffman), Mathematical Sciences Research Institute Publications 17 (Springer, Berlin, 1991), pp. 119-126.

18. M. L. LAPIDUS, 'Minkowski dimension and lattice points in homogeneously expanding domains with fractal boundary', in preparation.

19. M. L. LAPIDUS, 'Spectral and fractal geometry: from the Weyl-Berry conjecture for the vibrations of fractal drums to the Riemann zeta-function', Differential equations and mathematical physics, Proceedings of the UAB International Conference on Mathematical Physics and Differential Equations, Birmingham, Alabama, March 1990 (ed. C. Bennewitz, Academic Press, New York, 1992), pp. 151-182. 
20. M. L. LAPIDUS and J. Fleckinger-Pellé, 'Tambour fractal: vers une résolution de la conjecture de Weyl-Berry pour les valeurs propres du laplacien', C. R. Acad. Sci. Paris Sér. I Math. 306 (1988) 171-175.

21. M. L. LAPIDUS and H. MAIER, 'The Riemann hypothesis, inverse spectral problem for vibrating fractal strings and the modified Weyl-Berry conjecture', J. London Math. Soc. (2) to appear. Announced in 'Hypothèse de Riemann, cordes fractales vibrantes et conjecture de Weyl-Berry modifiée', C. R. Acad. Sci. Paris Sér. I Math. 313 (1991) 19-24.

22. M. L. Lapidus and C. Pomerance, 'Fonction zêta de Riemann et conjecture de Weyl-Berry pour les tambours fractals', C. R. Acad. Sci. Paris Sér. I Math. 310, No 6 (1990) 343-348.

23. J. L. LIONS and E. MAGENES, Non-homogeneous boundary value problems and applications, vol. I, English translation (Springer, Berlin, 1972).

24. B. B. MANDELBROT, The fractal geometry of nature, revised and enlarged edition (W. H. Freeman, New York, 1983).

25. O. MARTIO and M. VUORINEN, 'Whitney cubes, $p$-capacity, and Minkowski content', Exposition. Math. 5 (1987) 17-40.

26. R. B. MELROSE, 'Weyl's conjecture for manifolds with concave boundary', Geometry of the Laplace operator (eds R. Osserman and A. Weinstein), Proceedings of Symposia in Pure Mathematics 36 (American Mathematical Society, Providence, R. I., 1980), pp. 254-274.

27. R. B. Melrose, 'The trace of the wave group', Microlocal analysis (eds M. S. Baouendi, R. Beals, and L. P. Rothschild), Contemporary Mathematics 27 (American Mathematical Society, Providence, R. I., 1984), pp. 127-167.

28. G. MÉTIVIER, 'Valeurs propres de problèmes aux limites elliptiques irréguliers', Bull. Soc. Math. France, Mém. 51-52 (1977) 125-219.

29. The Lai Pham, 'Meilleures estimations asymptotiques des restes de la fonction spectrale et des valeurs propres relatifs au laplacien', Math. Scand. 48 (1981) 5-38.

30. R. T. SEElEy, 'A sharp asymptotic remainder estimate for the eigenvalues of the Laplacian in a domain of $\mathbb{R}^{3}$, Adv. in Math. 29 (1978) 244-269.

31. R. T. SEELEY, 'An estimate near the boundary for the spectral function of the Laplace operator', Amer. J. Math. 102 (1980) 869-902.

32. E. C. Titchmarsh, The theory of the Riemann zeta-function, 2 nd edn (revised by D. R. Heath-Brown, Oxford University Press, 1986).

33. C. Tricot JR, 'Douze définitions de la densité logarithmique', C. R. Acad. Sci. Paris Sér. I Math. 293 (1981) 549-552.

34. C. Tricot JR, 'Two definitions of fractal dimension', Math. Proc. Cambridge Philos. Soc. 91 (1982) 57-74.

35. H. WeYL, 'Über die Abhāngigkeit der Eigenschwingungen einer Membran von deren Begrenzung', J. Angew. Math. 141 (1912) 1-11.

\section{Department of Mathematics Sproul Hall \\ University of California Riverside California 92521-0135 U.S.A.}

\author{
Department of Mathematics \\ Boyd Graduate Studies Research Center \\ University of Georgia \\ Athens \\ Georgia 30602 \\ U.S.A.
}

